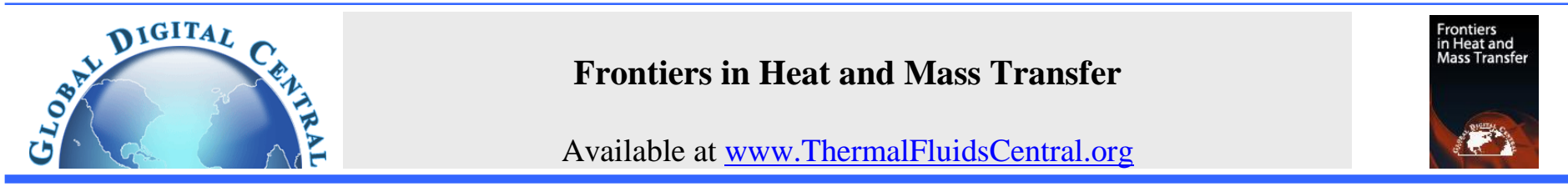

\title{
NEW SIMILARITY SOLUTION OF MICROPOLAR FLUID FLOW PROBLEM OVER AN UHSPR IN THE PRESENCE OF QUARTIC KIND OF AUTOCATALYTIC CHEMICAL REACTION
}

\author{
O. K. Koriko and I. L. Animasaun* \\ Department of Mathematical Sciences, Federal University of Technology, Akure, PMB 704, Nigeria
}

\begin{abstract}
The motion of air (i.e fluid) in which tiny particle rotates past a pointed surface of a rocket (as in space science), over a bonnet of a car and past a pointed surface of an aircraft is of important to experts in all these fields. Geometrically, all the domains of fluid flow in all these cases can be referred to as the upper horizontal surface of a paraboloid of revolution (uhspr). Meanwhile, the solution of the corresponding partial differential equation is an open question due to unavailability of suitable similarity variable to non-dimensionalize the angular momentum equation. This article unravels the nature of skin friction coefficient, heat transfer rate, velocity, temperature, concentration of homogeneous bulk fluid and heterogeneous catalyst which exists on a stretchable surface which is neither a perfect horizontal/vertical nor inclined/cone. Theory of similarity solution was adopted to obtain the similarity variable suitable to scale the proposed angular momentum equation. These equations along with the boundary conditions are solved numerically using Runge-Kutta technique along with shooting method. The similarity variable successfully nondimensionalized and parameterized the angular momentum for boundary layer flow past $u$ hspr. Temperature dependent dynamic viscosity parameter increases vertical velocity near a free stream but reduces micro-rotation near $u$ hspr. Effect of thermal radiation parameter on temperature profile and heat transfer rate can be greatly influenced by thickness parameter.
\end{abstract}

Keywords: Micropolar fluid, Paraboloid of revolution, Boundary layer analysis, Temperature dependent dynamic viscosity, Temperature dependent vortex viscosity.

\section{INTRODUCTION}

Due to the relevance of fluid flow in engineering, chemical and mechanical industries, space science and aviation; the analysis of different fluid flow within the thin boundary layer over various geometries have been investigated. Blasius (1908) and Sakiadis (1961) deliberated on the flow of a Newtonian fluid past a stretchable surface at the free stream and at the wall respectively. This area of interest in the field of fluid mechanics (boundary layer theory together with heat and mass transfer) has attracted the attention of Murphy (1953), Sowerby and Cooke (1953), Moore (1963), Sawchuk and Zamir (1992), Babu et al. (2015), Animasaun (2016), Motsa and Animasaun (2016a,b), Naramgari and Sulochana (2016), Sulochana et al. (2016) and Sandeep et al. (2016). Sandeep and Animasaun (2017) to deliberate on the boundary layer formed on spherical gas bubble, curved surface, cylinder, stagnation-point flow of a Carreau nanofluid towards a stretching/shrinking sheet, finite flat plate/sliding plate, wing of aircraft, impulsively started vertical porous surface, stagnation point flow of a micropolar fluid towards melting surface, 3-dimensional flow of Casson fluid towards a stagnation point at initial unsteady stage and final steady stage, nanofluid containing both nanoparticles and gyrotactic microorganisms due to impulsive motion flow of nanofluid, permeable stretching/shrinking sheet in the presence of suction/injection, inclined stationary/moving flat plate and over a wedge. In real life, there are many occurrences of either Newtonian fluid flow or non-Newtonian fluid flow (for instance air, nanofluid, water, Williamson fluid, micropolar fluid and Casson fluid etc.) past a pointed edge of a space shuttle, bonnet of a car and aircraft. It may not be realistic to refer to the surface of such object as vertical nor horizontal; likewise, neither inclined/wedge nor cone; hence the boundary layer formed on an upper horizontal surface of a paraboloid of revolution is very important. Considering the free convective flow of a nanofluid past an upper horizontal surface of a paraboloid of revolution, Animasaun (2016) explained the significance of the case in which gravity is sufficiently strong enough to make the specific weight appreciably different between any two layers of fluid. Recently, Makinde and Animasaun $(2016 a, b)$ focused on the flow of alumina-water nanofluid containing gyrotactic-microorganism over an upper horizontal surface of a paraboloid of revolution during the homogeneous-heterogeneous quartic autocatalytic chemical reaction in the absence and presence of thermophoresis together with the Brownian motion of $36 \mathrm{~nm}$ nanoparticles.

In fluid mechanics, it is a well-known fact that there exists no single fluid that can be referred to as a perfect Newtonian fluid. In the flow of non-Newtonian fluids, there exists no linear relationship between stress and deformation rate. The dynamics of non-Newtonian fluid with microstructure has become a popular area of research. One of the limitations of general Navier-Stokes equation is that it cannot be used to describe fluid flow with microstructure(s) (i.e. animal blood,

\footnotetext{
*Corresponding author.Email: anizakph2007@gmail.com.
} 
body fluids, lubricating oils and liquid crystals). However, each of the particle in this kind of fluids can rotate without depending on the motion of the fluid. In order to account for these behaviors in fluid flow, a theory which takes into account the micro-rotation and deformation are needed. The concept of simple microfluidic was started by Turkish-American engineering scientist Ahmed Cemal Eringen. According to the report of Eringen (1964), simple micro-fluid is a kind of liquid in which its properties and behaviors are influenced by the local motion of the fluid elements. Micropolar fluid can support body moments, stress moments and are influenced by the spin inertia. The stress tensor for these fluids is non-symmetric. Due to the complexity involved, the theory was simplified and a subclass of these fluids known as micropolar fluids was presented by Eringen (1966). In addition, Lukazewicz (1999) stated that micropolar fluid belongs to a class of fluids with non-symmetric stress tensor and consists of particles with a spinning motion. In summary, micropolar fluids can be defined as fluids consisting of rigid randomly oriented micro-rotations of particles suspended in a viscous medium. Moreover, Navier-Stoke equation is built on the assumption that material particle must possess three translational degrees of freedom. On the other side, material particle in micropolar fluids possesses three translational degrees of freedom together with another three rotational degrees of freedom. Gupta (2014) further explained that the three rotational degrees of freedom bring into existence non-symmetrical stress tensor and couple stresses; micro-rotations variables that represent spin and micro-inertia tensors which describe the distribution of atoms and molecules inside the microscopic fluid particles. Mohanty et al. (2015) investigated unsteady heat and mass transfer characteristics of a viscous incompressible electrically conducting micropolar fluid. This subject matter attracted Ojjela and Kumar (2016) to investigate the effects of hall and ion slip currents on an incompressible free convective flow, heat and mass transfer of a micropolar fluid in a porous medium between expanding or contracting walls with chemical reaction. It is worth mentioning that the behavior of this non-Newtonian fluid (micropolar) flow past the upper horizontal surface of a paraboloid of revolution is still an open question.

Transfer of heat energy can be described as the movement of temperature (heated particles) from hot objects to cold objects. Heat transfer can be grouped into three broad categories which are conduction, convection, and radiation. Pierre Prévost first explained Pictets experiment and argued extensively that all bodies (either hot or cold) radiate heat energy. Carinthia Slove Physicist, Mathematician, and Poet Josef Stefan presented an experimental measurement of total radiant heat energy in the year 1879. Thereafter, one of his Doctoral students (Ludwig Boltzmann) adopted the experimental results and theoretically derived what is known as Stefan-Boltzmann law. A pioneer in the field of theoretical astrophysics (Svein Rosseland) stated that when a medium is optically thick, the radiation can be approximated as an isotropic "diffusion" process; see Rosseland (1931). Perdikis and Raptis (1996) used a linearized form of the Rosseland approximation in the analysis of steady micropolar fluid flow past an unmoving plate. It is also important to remark that in most published articles on the effect of thermal radiation; Rosseland approximation has always been simplified by using Taylor series expansion and truncated the higher order terms. Consequently, the non-dimensionalization and parameterization of thermal radiation model become a simple task. Physically and scientifically, the electromagnetic radiation which occurs within the boundary layer of fluid as it flows during industrial processes may not be accurately studied using the so-called technique. In addition, the Taylor series expansion may not give full details on thermal radiation within boundary layer flows. In view of this, nonlinear thermal radiation has been a newly introduced model for accurate explanation. A comprehensive numerical analysis on the influences of nonlinear thermal radiation within boundary layer was presented by Cortell (2013). Hayat et al. (2015) and Animasaun et al. (2016) studied the effects nonlinear thermal radiation on the threedimensional nanofluid flow and viscoelastic fluid flow in the presence of induced magnetic field respectively. In the study of boundary layer analysis of micropolar fluid and unsteady magnetohydrodynamic Newtonian fluid flows through a binary mixture in an optically thin environment; Animasaun $(2015 ; 2015)$ considered the radiative heat flux term which models the thermal radiation in the flow as the fourth power of temperature in the energy equation. It is pertinent to notice that the dimensionless equation was obtained without using Taylor series expansion. However, an improved theoretical analysis is guaranteed if radiative heat flux is not expanded using Taylor series.

In all the above studies no attention has been given to investigate boundary layer flow of micropolar fluid over a paraboloid of revolution. In this present study, space dependent internal heat source model is included in the energy equation; and its corresponding influence on dynamic fluid viscosity, rotational velocity, and thermal conductivity are explored. The similarity solution of the governing equation is obtained using the first principle to obtain suitable similarity variable for scaling angular momentum equation governing micropolar fluid flow past uhspr. It is worth mentioning that the results of this present study will provide useful information to engineers in the industry, scientist who are involved in the production of rocket (space shuttle) and most especially to chemical engineers. In this article, the mathematical formulation of the problem is presented in section 2 while the numerical solution of the dimensionless equations using shooting technique along with classical Runge-Kutta Scheme is presented in section 3. In section 4, all the results are explained, and thereafter presented the conclusions based on the findings in section 5 .

\section{GOVERNING EQUATIONS}

In this section, the similarity solution of governing equations for micropolar fluid flow past $u$ hspr, the mathematical formulation which model the boundary layer flow past uhspr in the presence of space dependent internal heat source, nonlinear thermal radiation and quartic autocatalytic kind of homogeneous-heterogeneous chemical reaction are presented.

\subsection{Similarity variables for angular momentum}

Similarity solutions to Partial Differential Equations (PDEs) are solutions which depend on certain groupings of some variables. The computational constraints involved in solving PDEs is usually cumbersome, which makes it appropriate to seek for its advantageous and convenience in seeking a common solution in terms of dimensionless variables. In fluid dynamics, a similarity solution is a form of a solution which describes a flow that "looks the same" either at all times or at all length scales. It is then necessary to construct a length scale or time scale using space or time and other dimensional quantities which are present in the governing equation - such as characteristic length, kinematics viscosity and stretching velocity at the wall or velocity of the flow of the free stream. These constructs are not "guessed" but are derived from the scaling of the governing equations using the order of magnitude. Firstly, we assume that the velocity distribution within the boundary layer is similar to that of the dimensionless velocity known as $u / U_{o}$. Following the idea of Blasius (1908), the dimensionless distance from the wall " $\eta$ " is the ratio of distance in $y$-direction to the thickness of boundary layer

$\eta=\frac{y}{\delta}$ and $\frac{\delta}{x} \approx \operatorname{Re}_{x}^{-\frac{1}{2}}$ 
It is also assumed that within the boundary layer the usual balance between viscosity and convective inertia is struck, resulting in the scaling argument

$\frac{U_{o}^{2}}{L} \approx \frac{U_{o}}{\delta^{2}} \vartheta$

Here, $\vartheta$ is known as kinematic viscosity. And $L$ is known as characteristic length of the horizontal wall on upper horizontal surface of paraboloid of revolution; herein defined as

$L=\frac{2}{m+1}(x+b)^{-m+1}$

Substituting Eq. (3) into Eq. (2), we shall obtain

$\frac{U_{o}}{\frac{2}{m+1}(x+b)^{-m+1}} \approx \frac{1}{\delta^{2}} \vartheta$

From this scaling argument, it is apparent that the boundary layer grows with the downstream coordinate

$\delta \approx \sqrt{\frac{2}{m+1}} \sqrt{\frac{1}{(x+b)^{m-1}}} \sqrt{\frac{\vartheta}{U_{o}}}$

Substituting the boundary layer thickness Eq. (4) into the first term of Eq. (1)

$$
\eta=\frac{y}{\sqrt{\frac{2}{m+1}} \sqrt{\frac{1}{(x+b)^{m-1}}} \sqrt{\frac{\vartheta}{U_{o}}}}
$$

Hence, this suggests adopting the similarity variable

$\eta=y \sqrt{\frac{m+1}{2}}(x+b)^{\frac{m-1}{2}} \sqrt{\frac{U_{o}}{\vartheta}}$

together with

$\frac{u}{U_{o}}=(x+b)^{m} \frac{d f}{d \eta}$

By using Eq. (5) we can easily obtain Eq. (6) from the stream function which we herein defined as

$\psi(x, y)=\left(\frac{2}{m+1}\right)^{1 / 2}\left(\vartheta U_{o}\right)^{1 / 2}(x+b)^{\frac{m+1}{2}} f(\eta)$

In addition, similarity variables for angular momentum is of the form

$P\left(x, A[x+b]^{\frac{1-m}{2}}\right)=U_{o} \sqrt{\frac{m+1}{2}} \sqrt{\frac{U_{o}}{\vartheta}}(x+b)^{\frac{3 m-1}{2}} r(\eta)$

\subsection{Formulation of Governing Equation}

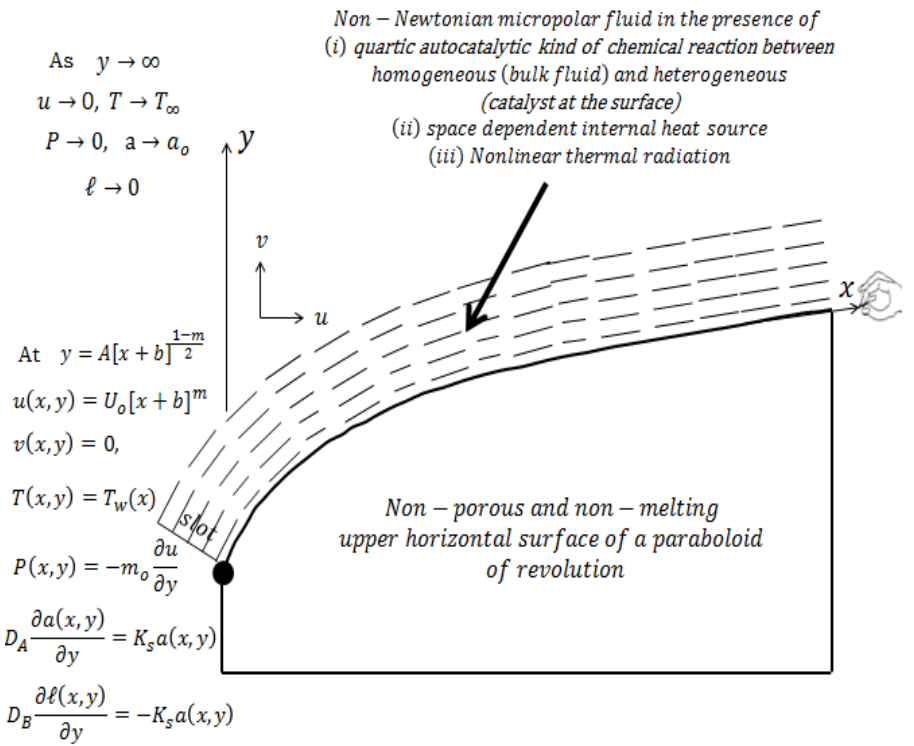

Fig. 1 The coordinate system of micropolar fluid flow past $u$ hspr.
Steady two-dimensional micropolar (non-Newtonian) fluid flow past an upper horizontal surface of a paraboloid of revolution in the presence of homogeneous-heterogeneous chemical reaction is considered. In the micropolar fluid, it is assumed that quartic autocatalytic chemical reaction occurs between homogeneous bulk fluid (species $A$ ) and heterogeneous catalyst on uhspr (species $B$ ) as it flows. The chemical reaction can be described as a kind in which the homogeneous (bulk fluid) reaction is assumed to be by isothermal quartic autocatalytic kinetics and the heterogeneous (catalyst at the surface) reaction is assumed to be by first order kinetics. The concentration of homogeneous bulk fluid (micropolar) $A$ is " $a$ ". The concentration of catalyst at the surface is " $\ell$ ". The micropolar fluid flow under consideration is assumed to occupy the domain $A(x+b)^{\frac{1-m}{2}} \leq y<\infty$ as shown in Fig. 1. The immediate fluid layers on $u$ hspr are stretched parallel with velocity $U_{w}=U_{o}(x+b)^{m}$. The paraboloid of revolution is assumed to be non-porous and non-melting. In this case, $x$-axis is taken along the direction of the horizontal surface and $y$-axis is normal to it. The origin of $x$-axis and $y$-axis are not the starting point of the fluid flow but is a function of the form $y=A(x+b)^{\frac{1-m}{2}}$ where the parameter $m$ is known as velocity power index and $b$ is known as a parameter related to the stretching sheet. Consequently, the velocity along $x$-direction, velocity along $y$-direction, temperature, microrotation, concentration of species $A$ and concentration of species $B$ at the surface are $u(x, y), v(x, y), T(x, y), \quad \mathrm{P}(x, y), \quad a(x, y)$ and $\ell(x, y)$ respectively. Following the formulation of Chaudhary and Merkin $(1995 ; 1996)$ the governing boundary-layer equation can be expressed as

$\frac{\partial u}{\partial x}+\frac{\partial v}{\partial y}=0$,

$u \frac{\partial u}{\partial x}+v \frac{\partial u}{\partial y}=\left(\frac{\mu+\tau}{\rho}\right) \frac{\partial^{2} u}{\partial y^{2}}+\frac{\tau}{\rho} \frac{\partial P}{\partial y}+g \beta \frac{m+1}{2}\left(T-T_{\infty}\right)$.

The temperature at the free stream $\left(T_{\infty}\right)$ is a constant function at all points in $x$-direction. Considering the influences of wall temperature $T_{w}$ and space dependent internal heat source, it is assumed that the dynamic viscosity of the micropolar fluid vary linearly with temperature while vortex viscosity of the micropolar fluid varies exponentially with temperature. These assumptions are based on the correlation and nature of rotation of microconstituent in form of vortex. Modified momentum equation for non-Newtonian micropolar fluid

$u \frac{\partial u}{\partial x}+v \frac{\partial u}{\partial y}=\frac{\partial}{\partial y}\left(\mu(T) \frac{\partial u}{\partial y}\right)+\frac{\tau(T)}{\rho} \frac{\partial^{2} u}{\partial y^{2}}+\frac{\tau(T)}{\rho} \frac{\partial P}{\partial y}+g \beta \frac{m+1}{2}\left(T-T_{\infty}\right)$.

In the micropolar fluid, radiative transport equation (RTE) for the case of optically thick media is adopted to account for the radiative heat flux as

$q_{r}=-\frac{4 \sigma^{*}}{3 k^{*}} \frac{\partial T^{4}}{\partial y}$

where $\sigma^{*}$ and $k^{*}$ are the Stefan-Boltzman constant and the mean absorption coefficient respectively. It is assumed that the temperature difference between the layers of micropolar fluid flow is not sufficiently small. In view of this, it may not be realistic to simplify the radiative heat flux by expanding $T^{4}$ in a Taylor series expansion about $T_{\infty}$ then neglecting higher order terms. Using implicit differentiation to evaluate the partial rate of change of $T^{4}$ with $y$ in Eq. (9) and then substitute into energy equation leads to The energy equation in which thermal radiation is accounted for is of the form

$$
\begin{aligned}
& u \frac{\partial T}{\partial x}+v \frac{\partial T}{\partial y}=\frac{1}{\rho c_{p}} \frac{\partial}{\partial y}\left(\kappa(T) \frac{\partial T}{\partial y}\right)+\frac{16 \sigma^{*}}{3 k^{*} \rho c_{p}} T^{3} \frac{\partial^{2} T}{\partial y^{2}}+\frac{16 \sigma^{*}}{3 k^{*} \rho c_{p}} \frac{\partial T}{\partial y} \frac{\partial T^{3}}{\partial y}+ \\
& +\frac{Q_{o}\left(T_{w}-T_{\infty}\right)}{\rho c_{p}} \mathrm{e}^{-n y \sqrt{\frac{m+1}{2}}(x+b)^{\frac{m-1}{2}} \sqrt{\frac{U_{o}}{g}}}
\end{aligned}
$$


Substituting temperature ratio parameter $\theta_{w}=T_{w} / T_{\infty}$ into the similarity variable for temperature $\theta=\frac{T-T_{w}}{T_{w}-T_{\infty}}$ due to the fact that it cannot be used to successfully parameterize and non-dimensionalize the terms $T^{3}$ and $\frac{\partial T^{3}}{\partial y}$ in Eq. (13). Leads to

$T=T_{\infty}\left[1+\theta \theta_{w}-\theta\right]$

Adopting the model for homogeneous-heterogeneous reactions by Chaudhary and Merkin (1995) together with Lynch (1992), within the boundary layer we propose isothermal Nth autocatalytic reaction of the form

$A+N B \rightarrow(\mathrm{N}+1) \mathrm{B}$

the rate of chemical reaction $=k_{1} a \ell^{N}$ and on the upper horizontal surface of a paraboloid of revolution in the presence of a catalyst, there exists single isothermal first order reaction of the form

$A \rightarrow \mathrm{B}$

rate of chemical reaction $=k_{s} a$ where " $a$ " and " $\ell "$ are the concentrations of reactant $\mathrm{A}$ and reactant $\mathrm{B}$. Here, $k_{1}$ and $k_{s}$ are known as the reaction rate coefficient which may not be actually referred to as a constant because it includes all the likely parameters that may affect reaction rate except concentration which has been explicitly accounted for in Eq. (15) and Eq. (16). However, setting $\mathrm{N}=3$, the schemes (i.e. Eq. (15) and Eq. (16)) corresponds with that of quartic autocatalytic kind of chemical reaction. In order to accurately study the behavior of the micropolar fluid flow past the upper surface of a horizontal paraboloid of revolution, the addition of gyration vector and fluid shear (angular velocity) is proposed as a multiple of the ratio of stretching acceleration to stretching rate. Angular momentum equation suitable to model micropolar fluid flow along an upper horizontal surface of a paraboloid of revolution is

$u \frac{\partial P}{\partial x}+v \frac{\partial P}{\partial y}=\frac{\gamma^{*}}{\rho j} \frac{\partial^{2} P}{\partial y^{2}}-\frac{\tau}{\rho j U_{o}} \frac{\partial U_{w}}{\partial x}\left(2 P+\frac{\partial u}{\partial y}\right)$.

Homogeneous-heterogeneous reaction model for the concentrations of chemical species $A$ and $B$, as stated in the reaction scheme Eq. (17) and Eq. (16)

$$
\begin{aligned}
& u \frac{\partial a}{\partial x}+v \frac{\partial a}{\partial y}=D_{A} \frac{\partial^{2} a}{\partial y^{2}}-K_{1} a \ell^{N} \\
& u \frac{\partial \ell}{\partial x}+v \frac{\partial \ell}{\partial y}=D_{B} \frac{\partial^{2} \ell}{\partial y^{2}}+K_{1} a \ell^{N}
\end{aligned}
$$

Considering the fact that reactant $\mathrm{A}$ and reactant $\mathrm{B}$ undergo chemical changes at the interface, the heterogeneous catalytic reaction is properly accounted for. In this study, the boundary condition of micropolar fluid at most minimum level (immediate fluid layer on an upper horizontal surface of a paraboloid of revolution) is $P(x, y)$. In the absence of suction/injection, Eq. (9), Eq. (11), Eq. (14), Eq. (17), Eq. (18) and Eq. (19) are subject to the following boundary conditions

$$
\begin{gathered}
u=U_{o}(x+b)^{m}, \quad v=0, \quad T=T_{w}, \quad P=-m_{o} \frac{\partial u}{\partial y}, \quad \frac{\partial a}{\partial y}=\frac{k_{s} a}{D_{A}}, \\
\frac{\partial \ell}{\partial y}=-\frac{k_{s} a}{D_{B}} \text { at } y=A(x+b)^{\frac{1-m}{2}}
\end{gathered}
$$

$u \rightarrow 0, \quad T \rightarrow T_{\infty}, \quad P \rightarrow 0, \quad a \rightarrow a_{o}, \quad \ell \rightarrow 0 \quad$ as $\quad y \rightarrow \infty$

Considering the experimental data presented by Batchelor (1987) which describes the variation of dynamic viscosity with temperature and this leads to temperature dependent viscosity model adopted by Mukhopadhyay (2009) as in Eq. (22a). Following Charraudeau (1975), the mathematical model for temperature dependent thermal conductivity is of the form Eq. (22b). Considering the flow of micropolar fluid over a heated upper horizontal catalytic surface of a paraboloid of revolution, it is assumed that the vortex viscosity varies exponentially with temperature. Reynold's model as in Equation (22c) is adopted.

$$
\begin{array}{ll}
\mu(T) & =\mu^{*}\left[1+j_{1}\left(T_{w}-T\right)\right], \\
\kappa(T) & =\kappa^{*}\left[1+j_{2}\left(T-T_{\infty}\right)\right],
\end{array}
$$

All the models stated in Eq. (22) are valid since $T_{w}>T_{\infty}$. The quantities of interest are the skin friction coefficient $C f$ and Nusselt number $N u_{x}$ which are defined as

$$
C f=\frac{\tau_{w}}{\rho\left(U_{w}\right)^{2} \sqrt{\frac{m+1}{2}}} \quad N u_{x}=\frac{(x+b) q_{w}}{\kappa\left[T_{w}(x)-T_{\infty}\right] \sqrt{\frac{m+1}{2}}}
$$

where $\tau_{w}$ is known as Shear stress or skin friction along the stretching sheet along the upper surface of a horizontal paraboloid of revolution and $q_{w}$ is known as heat flux from the upper surface of a horizontal paraboloid of revolution

$$
\tau_{w}=\left.\mu \frac{\partial u}{\partial y}\right|_{y=A[x+b]^{\frac{1-m}{2}}}, \quad q_{w}=-\left.\kappa \frac{\partial T}{\partial y}\right|_{y=A[x+b]^{\frac{1-m}{2}}}
$$

\subsection{Parameterization and Non-dimensionalization}

The transformation and non-dimensionalization of governing equations can be easily achieved by using the similarity variables and stream function defined in subsection 2.1 together with

$$
\begin{aligned}
& T_{w}(x)=A(x+b)^{\frac{1-m}{2}}, \quad \theta(\eta)=\frac{T-T_{\infty}}{T_{w}(x)-T_{\infty}}, \quad S_{c b}=\frac{\vartheta}{D_{B}}, \quad h(\eta)=\frac{\ell}{a_{o}} \\
& \aleph=\frac{\tau^{*}}{\mu}, \quad G r_{m}=\frac{g \beta}{j_{1} U_{o}^{2}(x+b)^{2 m-1}}, \quad \varepsilon=j_{2}\left[T_{w}(x)-T_{\infty}\right], \quad \theta_{w}=\frac{T_{w}(x)}{T_{\infty}},(25) \\
& g(\eta)=\frac{a}{a_{o}}, P_{r}=\frac{c_{p} \mu}{\kappa^{*}}, \quad R_{a}=\frac{3 k^{*} \kappa^{*}}{16 \sigma^{*} T_{\infty}^{3}}, \quad S_{c a}=\frac{\vartheta}{D_{A}}, \quad \zeta=b_{s}\left[T_{w}(x)-T_{\infty}\right], \\
& \gamma=\frac{Q_{o}}{\rho c_{p} U_{o}(x+b)^{m-1}}, K=\frac{K_{1} a_{o}^{N}}{U_{o}(x+b)^{m-1}}, \Lambda=\frac{K_{s}}{D_{A} \sqrt{\frac{m+1}{2}} \frac{U_{o}}{\vartheta}(x+b)^{\frac{m-1}{2}}}
\end{aligned}
$$

where $\aleph$ is known as micro-rotation parameter, $G r_{m}$ is known as buoyancy parameter, $\varepsilon$ is known as temperature-dependent thermal conductivity, $\theta_{w}$ is known as temperature ratio parameter, $\gamma$ is known as space dependent parameter, $P_{r}$ is known as Prandtl number, $R_{a} \mathrm{Ra}$ is known as radiation parameter, $\zeta$ is known as temperature-dependent vortex viscosity parameter, $S_{c a}$ is the ratio of viscous diffusion rate $(\mu)$ to molecular (mass) diffusion rate $\left(\rho D_{A}\right), S_{c b}$ is the ratio of viscous diffusion rate $(\mu)$ to molecular (mass) diffusion rate $\left(\rho D_{B}\right), K$ denotes strength of the homogeneous reaction, $\chi$ denotes thickness parameter and $\Lambda$ denotes strength of heterogeneous parameter we obtain dimensionless governing equation of the form

$$
\begin{gathered}
\left(1+\xi-\xi \theta+\aleph e^{-\theta \zeta}\right) \frac{d^{3} f}{d \eta^{3}}-\xi \frac{d \theta}{d \eta} \frac{d^{2} f}{d \eta^{2}}+f \frac{d^{2} f}{d \eta^{2}}-\frac{2 m}{m+1} \frac{d f}{d \eta} \frac{d f}{d \eta}+ \\
\aleph e^{-\theta \zeta} \frac{d r}{d \eta}+\xi G r_{m} \theta=0, \\
\left(1+\varepsilon \theta+\frac{\left(1+\theta \theta_{w}-\theta\right)^{3}}{R_{a}}\right) \frac{d^{2} \theta}{d \eta^{2}}+\varepsilon \frac{d \theta}{d \eta} \frac{d \theta}{d \eta}+P_{r} f \frac{d \theta}{d \eta}-P_{r} \frac{1-m}{m+1} \theta \frac{d f}{d \eta}+ \\
\frac{2 \gamma P_{r}}{m+1} e^{-n \eta}+\frac{3\left(1+\theta \theta_{w}-\theta\right)^{2}}{R_{a}}\left(\theta_{w}-1\right) \frac{d \theta}{d \eta} \frac{d \theta}{d \eta}=0,
\end{gathered}
$$




$$
\begin{aligned}
& \left(1+\xi-\xi \theta+\frac{\aleph e^{-\theta \zeta}}{2}\right) \frac{d^{2} r}{d \eta^{2}}-\frac{3 m-1}{m+1} r \frac{d f}{d \eta}+ \\
& \quad f \frac{d r}{d \eta}-\frac{\aleph e^{-\theta \zeta}}{[1+\xi-\xi \theta]} \frac{2 m}{m+1}\left(2 r+\frac{d^{2} f}{d \eta^{2}}\right)=0 \\
& \frac{d^{2} g}{d \eta^{2}}+S_{c a} f \frac{d g}{d \eta}-S_{c a} K \frac{2}{m+1} g h^{N}=0 \\
& \delta \frac{d^{2} h}{d \eta^{2}}+S_{c b} f \frac{d h}{d \eta}+S_{c b} K \frac{2}{m+1} g h^{N}=0
\end{aligned}
$$

It is pertinent to note that the minimum value of $y$ is not the starting point of the slot. This implies that all the conditions in Eq. (20) are not imposed at $y=0$ but at $y=A(x+b)^{\frac{1-m}{2}}$. As shown in Fig. 1 and Fig. 2 , it is obvious that it may not be realistic to say that $y=0$ at all points on the upper horizontal surface of a paraboloid of revolution. Hence, not valid to set $y=0$ in similarity variable $\eta$. Upon using $y=A(x+b)^{\frac{1-m}{2}}$ (i.e. the starting point of the flow at the slot), minimum value of $y$ which accurately corresponds to minimum value of similarity variable $\eta$ as

$$
\eta=A\left(\frac{m+1}{2} \frac{U_{o}}{\vartheta}\right)^{1 / 2}=\chi
$$

This implies that at the wall the boundary condition suitable to scale the boundary layer flow is $\eta=\chi$. The boundary condition becomes

$$
\begin{gathered}
\frac{d f}{d \chi}=1, \quad f(\chi)=\chi \frac{1-m}{m+1}, \quad \theta(\chi)=1, \quad r=-m_{o} \frac{d^{2} f}{d \eta^{2}}, \quad \frac{d g}{d \chi}=\Lambda g(\chi), \\
\delta \frac{d h}{d \chi}=-\Lambda g(\chi) \quad \text { at } \quad \eta=\chi
\end{gathered}
$$$$
\frac{d f}{d \chi} \rightarrow 1, \quad \theta(\chi) \rightarrow 0, r(\chi) \rightarrow 0, \quad g(\chi) \rightarrow 1, h(\chi) \rightarrow 0, \text { as } \eta \rightarrow \chi
$$

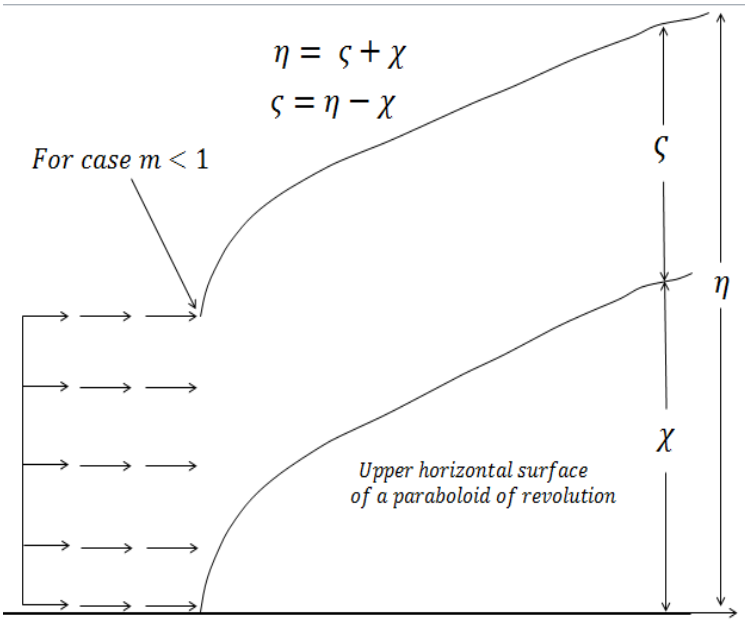

Fig. 2 Graphical illustration of fluid domain and conversion of domain from $\eta \in[\chi, \infty)$ to $\varsigma \in[0, \infty)$

Moreover, dimensionless governing Eq. (26) to Eq. (30) are depending on $\eta$ while the boundary conditions Eq. (31) and Eq. (32) are functions and/or derivatives depending on $\chi$. In order to transform the domain from $\eta \in[\chi, \infty)$ to $\varsigma \in[0, \infty)$ it is valid to adopt

$F(\varsigma)=F(\eta-\chi)=f(\eta), \Theta(\varsigma)=\Theta(\eta-\chi)=\theta(\eta), R(\varsigma)=R(\eta-\chi)$

$=r(\eta), G(\varsigma)=G(\eta-\chi)=g(\eta)$, and $H(\varsigma)=H(\eta-\chi)=h(\eta)$.
For more details see Fig. 2. The final dimensionless governing equation (coupled system of nonlinear ordinary differential equation) of the form

$$
\begin{aligned}
& \left(1+\xi-\xi \Theta+\aleph e^{-\Theta \zeta}\right) \frac{d^{3} F}{d \varsigma^{3}}-\xi \frac{d \Theta}{d \varsigma} \frac{d^{2} F}{d \varsigma^{2}}+F \frac{d^{2} F}{d \varsigma^{2}}-\frac{2 m}{m+1} \frac{d F}{d \varsigma} \frac{d F}{d \varsigma}+ \\
& \aleph e^{-\Theta \zeta} \frac{d R}{d \varsigma}+\xi G r_{m} \Theta=0, \\
& \left(1+\varepsilon \Theta+\frac{\left(1+\Theta \theta_{w}-\Theta\right)^{3}}{R_{a}}\right) \frac{d^{2} \Theta}{d \varsigma^{2}}+\varepsilon \frac{d \Theta}{d \varsigma} \frac{d \Theta}{d \varsigma}+P_{r} F \frac{d \Theta}{d \varsigma}-P_{r} \frac{1-m}{m+1} \Theta \frac{d F}{d \varsigma}+ \\
& \frac{2 \gamma P_{r}}{m+1} e^{-n \varsigma}+\frac{3\left(1+\Theta \theta_{w}-\Theta\right)^{2}}{R_{a}}\left(\theta_{w}-1\right) \frac{d \Theta}{d \varsigma} \frac{d \Theta}{d \varsigma}=0 \\
& \left(1+\xi-\xi \Theta+\frac{\aleph e^{-\Theta \zeta}}{2}\right) \frac{d^{2} R}{d \varsigma^{2}}-\frac{3 m-1}{m+1} R \frac{d R}{d \varsigma}+ \\
& f \frac{d R}{d \varsigma}-\frac{\aleph e^{-\Theta \zeta}}{[1+\xi-\xi \Theta]} \frac{2 m}{m+1}\left(2 R+\frac{d^{2} F}{d \varsigma^{2}}\right)=0 \\
& \frac{d^{2} G}{d \varsigma^{2}}+S_{c a} F \frac{d G}{d \varsigma}-S_{c a} K \frac{2}{m+1} G H^{N}=0 \\
& \delta \frac{d^{2} H}{d \varsigma^{2}}+S_{c b} F \frac{d H}{d \varsigma}+S_{c b} K \frac{2}{m+1} G H^{N}=0 \\
& \text { Subject to } \\
& \frac{d F}{d \varsigma}=1, \quad F(\varsigma)=\chi \frac{1-m}{m+1}, \quad \Theta(\varsigma)=1, \quad R=-m_{o} \frac{d^{2} F}{d \varsigma^{2}}, \quad \frac{d G}{d \varsigma}=\Lambda G(\varsigma), \\
& \delta \frac{d H}{d \varsigma}=-\Lambda G(\varsigma) \quad \text { at } \quad \varsigma=0 \\
& \frac{d F}{d \varsigma} \rightarrow 1, \Theta(\chi) \rightarrow 0, R(\chi) \rightarrow 0, G(\varsigma) \rightarrow 1, H(\varsigma) \rightarrow 0, \text { as } \varsigma \rightarrow \chi
\end{aligned}
$$

Upon substituting the similarity variables Eq. (25) into Eq. (23) and Eq. (24) we obtain

$$
\operatorname{Re}_{x}^{1 / 2} C f=F^{\prime \prime}(0), \quad \mathrm{Nu}_{x} \operatorname{Re}_{x}^{1 / 2}=-\Theta^{\prime}(0)
$$

\section{NUMERICAL SOLUTION}

Numerical solution of corresponding boundary value problem Eqs. (33) - (37) subject to boundary condition (38) and (39) are obtained using classical Runge-Kutta method along with shooting techniques and MATLAB package $(b v p 5 c)$. The boundary value problem cannot be solved on an infinite interval and it would be impractical to solve it for even a very large finite interval. However, infinite boundary condition at a finite point is considered as $\varsigma=4$. The set of coupled nonlinear ordinary differential equations along with boundary conditions have been reduced to a system of seven simultaneous equations of the first order for seven unknowns following the method of superposition in $\mathrm{Na}$ (1979).

\subsection{Classical Runge-Kutta method along with shooting techniques}

In order to integrate the corresponding initial value problem, the values of $F^{\prime \prime}(0), \Theta^{\prime}(0), R^{\prime}(0), G^{\prime}(0)$, and $H^{\prime}(0)$ are required. However, such values do not exist after the non-dimensionalization of the boundary conditions Eq. 20 - Eq. 21. Although, at a specific value of $\delta$ and $\Lambda$ correct estimates for $G^{\prime}(0)$, and $H^{\prime}(0)$ can be easily obtained once $G(0)$, is known. The suitable guess values for $F^{\prime \prime}(0), \Theta^{\prime}(0)$, $R^{\prime}(0), G^{\prime}(0)$, and $H^{\prime}(0)$ are chosen and then numerical integration was carried out. The calculated values of $F^{\prime}(\varsigma), \Theta(\varsigma), R(\varsigma), G(\varsigma)$, 
and $H(\varsigma)$, at infinity ( $\varsigma=4)$ are compared with the given boundary conditions in Eq. (39) and the estimated values $F^{\prime \prime}(0), \Theta^{\prime}(0)$, $R^{\prime}(0), G^{\prime}(0)$, and $H^{\prime}(0)$ are adjusted to give a better approximation for the solution. Series of values for $F^{\prime \prime}(0), \Theta^{\prime}(0), R^{\prime}(0), G^{\prime}(0)$, and $H^{\prime}(0)$ are considered and applied with fourth-order classical RungeKutta method using step size $\Delta \varsigma=0.01$. The above procedure is repeated until asymptotically converged results are obtained within a tolerance level of $10^{-5}$. It is very important to remark that using $\varsigma_{\infty}=4$, all profiles are compatible with the boundary layer theory and asymptotically satisfies the conditions at free stream as suggested by Pantokratoras (2009). It is noteworthy that there exist no related published articles that could be used to validate the accuracy of the present numerical results. Meanwhile, Eq. (33) - Eq. (37) subject to boundary conditions Eq. 38 - Eq. 39 can easily be solved using ODE solvers such as MATLAB's $b v p 5 c$. Meanwhile, this boundary value problem can easily be solved using ODE solvers such as MATLAB's $b v p 5 c$; see Gökhan (2011) and Kierzenka and Shampine (2001).

\section{DISCUSSION OF RESULTS}

Dimensionless non-linear coupled differential equations together with Neumann boundary conditions are solved numerically using the method discussed in subsection 3.1. All the graphical results are presented using [Azimuth +90, Elevation -90]. The main purpose is to achieve better graphical profiles of $29 \mathrm{~nm} \mathrm{CuO}$-water nanofluid as it flows from left to right over the upper horizontal surface of a paraboloid of revolution. In addition, to achieve unequal diffusivities following the explanation of Chaudhary and Merkin (1995), $\delta=1.2, S_{c a}=0.62$, and $S_{c b}=1.3$, (i.e. $S_{c a} \neq S_{c b}$ ). The numerical computations have been carried out for various values of all parameters in order to study its effects on the motion of micropolar fluid within the boundary layer.

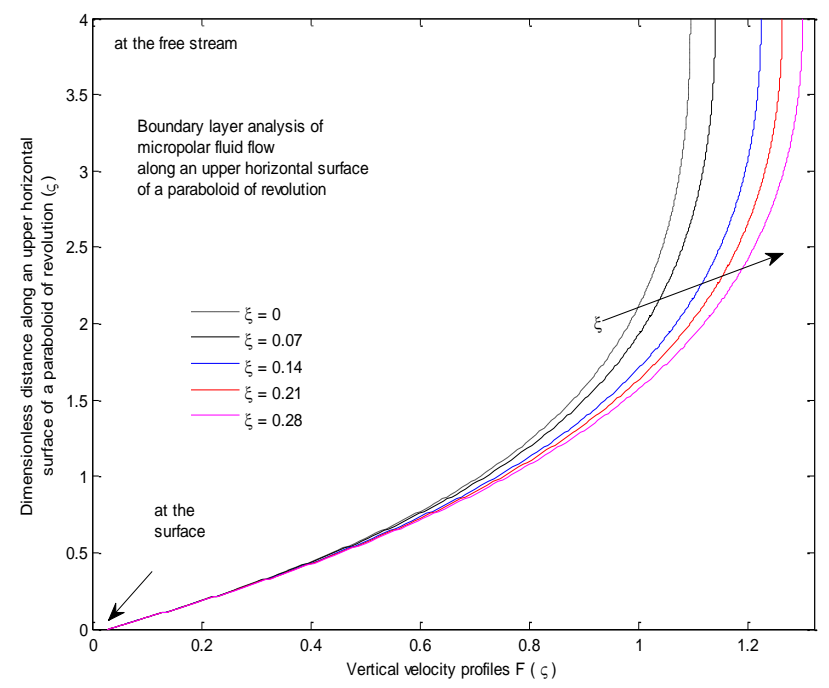

Fig. 3 Effect of $\xi$ on $F(\varsigma)$

The effects of all the parameters on the vertical velocity profiles $F(\varsigma)$, horizontal velocity profiles $F^{\prime}(\varsigma)$, temperature profiles $\Theta(\varsigma)$, temperature gradient profiles $\Theta^{\prime}(\varsigma)$, microrotation profiles $R(\varsigma)$, micro-rotation gradient profiles $R^{\prime}(\varsigma)$, concentration of reactant A (homogeneous - bulk fluid) $G(\varsigma)$, , concentration gradient of reactant A $G^{\prime}(\varsigma)$, concentration of reactant B (heterogeneous - catalyst at the surface) $H(\varsigma)$, and concentration gradient of reactant $\mathrm{B} H^{\prime}(\varsigma)$, within boundary layer flow of micropolar fluid are illustrated.

\subsection{Effects of temperature dependent dynamic viscosity parameter $(\xi)$ and temperature dependent vortex viscosity parameter $(\zeta)$ on the flow of micropolar fluid within boundary layer formed on uhspr}

Graphical illustrations of some profiles when $\aleph=0.4, m=0.3$, $\varepsilon=0.2, \operatorname{Pr}=0.7, \quad S_{c a}=0.62, \quad S_{c b}=1.3, \quad R=0.1, \theta_{w}=0.2, m_{o}=0.5$, $G_{r m}=1, K=0.05, \gamma=0.2, \mathrm{n}=0.3, \zeta=0.1, \chi=0.05, \Lambda=0.05$,

$\delta=1.2$ and $N=3$ at various values of $\xi$ are illustrated in Fig. 3 - Fig. 7. It is observed that vertical and horizontal velocity increases with $\xi$. The graph of shear stress profile reveals that there exist dual effects of $\xi$ on all the layers of fluids within the interval $0 \leq \varsigma \leq 4$. Meanwhile, it is certain that $F^{\prime \prime}(0)$ increases with $\xi$. Also, a negligible decrease in shear stress at free stream is observed. It is noticed that increasing effect of $\xi$ on temperature and temperature gradient is negligible, hence not presented for brevity. Micro-rotation profiles $R(\varsigma)$ are found to be a decreasing function of fluid layers near the upper horizontal surface of a paraboloid of revolution. The effect of temperature dependent viscosity parameter $(\xi)$ on micro-rotation gradient is illustrated in Fig. 7. It is seen that the micro-rotation gradient function $R^{\prime}(\varsigma)$ increases with $\xi$ near an $u$ hspr with no effect on fluid layers near a free stream. At the quartic autocatalytic level of chemical reaction $(N=3)$, it is observed that concentration of homogeneous bulk fluid increases while the concentration of heterogeneous catalyst at the surface decreases with $\xi$. For brevity, the graphical illustrations of $G(\varsigma)$ and $H(\varsigma)$ at various values of $\xi$ are not displayed. Variation in local skin friction coefficient $\operatorname{Re}_{x}^{1 / 2} C f$ at various values of buoyancy parameter $G_{r m}$ and temperature dependent dynamic viscosity parameter $\xi$ is presented in Fig. 8. It is noticed that $\operatorname{Re}_{x}^{1 / 2} C f$ decreases negligible with $\xi$ in the absence of buoyancy (i.e. $G_{r m}=0$ ). A significant increase in $\operatorname{Re}_{x}^{1 / 2} C f$ with $\xi$ is observed when $G_{r m}=1$. However, maximum local skin friction coefficient is guaranty when magnitudes of $G_{r m}$ and $\xi$ are large; see Fig. 8 more details. Using the set of all parameters above and $\xi=0.35, \aleph=4$ and $G_{r m}=1$, effects of $\zeta$ are illustrated in Fig. 9 - Fig. 16. It is observed that vertical velocity profiles decrease near a free stream while horizontal velocity decreases significantly within the fluid domain. Also, the microrotation profiles $R(\varsigma)$ increase near the wall with a negligible decrease thereafter till free stream. It is further observed that micro-rotation gradient $R^{\prime}(\varsigma)$ decreases near the wall and increases near a free stream. Vorticity is a word which describes the local spinning motion of tiny particles at a point and traveling along in the fluid flow. Physically, as the magnitude of $\zeta$ increases, the rate at which tiny particle rotates is increased due to the fact that vortex of the micropolar fluid is assumed to vary exponentially with temperature. This explains the decrease we observed in vertical and horizontal velocity with $\zeta$. Meanwhile, Fig. 11 confirms that the tiny particles actually rotate significantly near the wall. It is observed in Fig. 13 and Fig. 14 that concentration of the homogeneous bulk fluid decreases and heterogeneous catalyst at the wall increases with $\zeta$. When $\aleph=2$, $m=0.3, \quad \varepsilon=0.2, \operatorname{Pr}=0.7, \quad S_{c a}=0.62, \quad S_{c b}=1.3, \quad R=0.1, \quad \theta_{w}=0.2$, $m_{o}=0.5, \quad G_{r m}=1, \quad K=0.05, \quad \mathrm{n}=0.3, \quad \chi=0.05, \quad \Lambda=0.05, \quad \delta=1.2$ and $N=3$ variations in $F^{\prime \prime}(0)$, and $R(0)$, with both $\xi$ and $\zeta$ in the absence of space dependent heat source and in the presence of substantial internal heat source are presented in Table cc. It is observed that at fixed value of $\zeta$, local skin friction coefficients $\operatorname{Re}_{x}^{1 / 2} C f$ increases more significant with $\xi$ when $\gamma=0$ to compare to the case when $\gamma=1$. This fact is established by estimating linear regression line through data points $S l_{p}$ in $\operatorname{Re}_{x}^{1 / 2} C f$ and $\xi$ when $\gamma=0$ and $\gamma=1$ 
respectively. $S l_{p}$ when $\gamma=0$ is estimated as 3.14 and $\gamma=1$ is estimated as 1.61 .

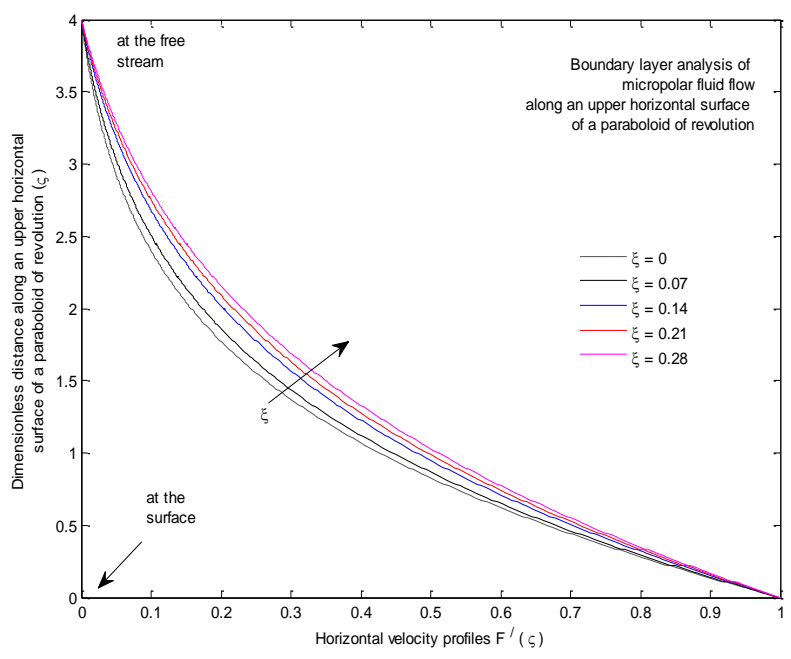

Fig. 4 Effect of $\xi$ on $F^{\prime}(\varsigma)$

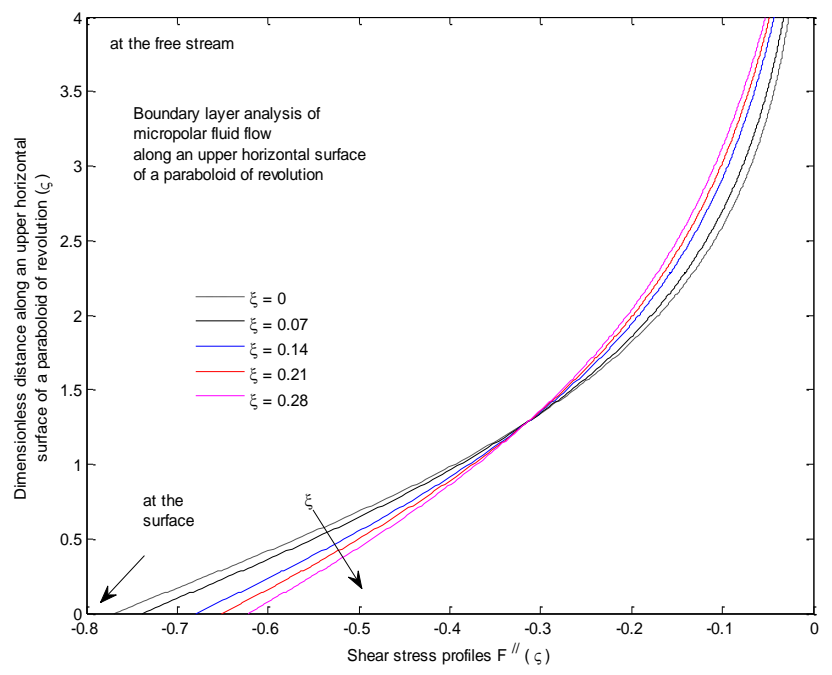

Fig. 5 Effect of $\xi$ on $F^{\prime \prime}(\varsigma)$

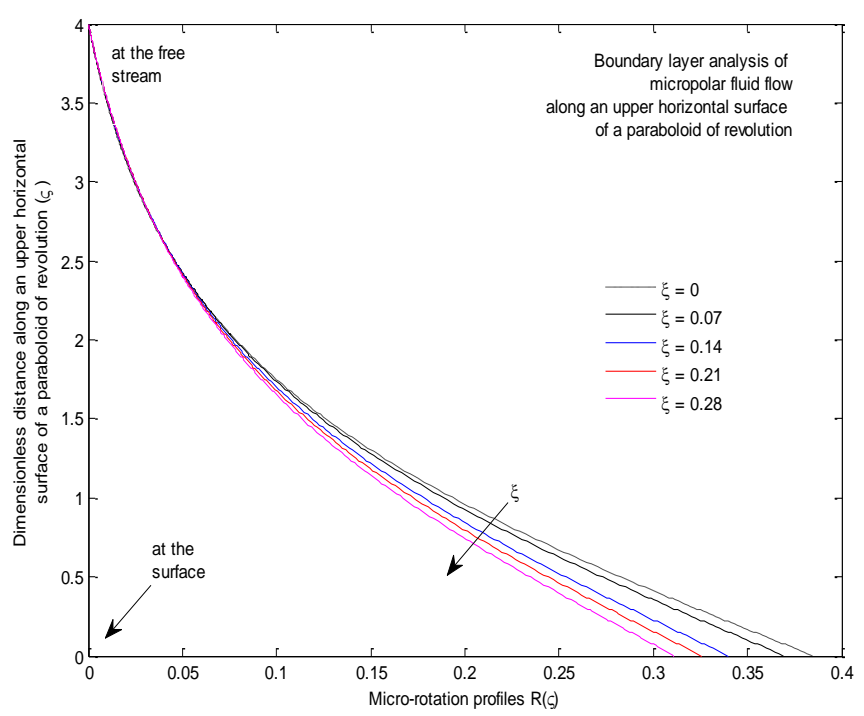

Fig. 6 Effect of $\xi$ on $R(\varsigma)$

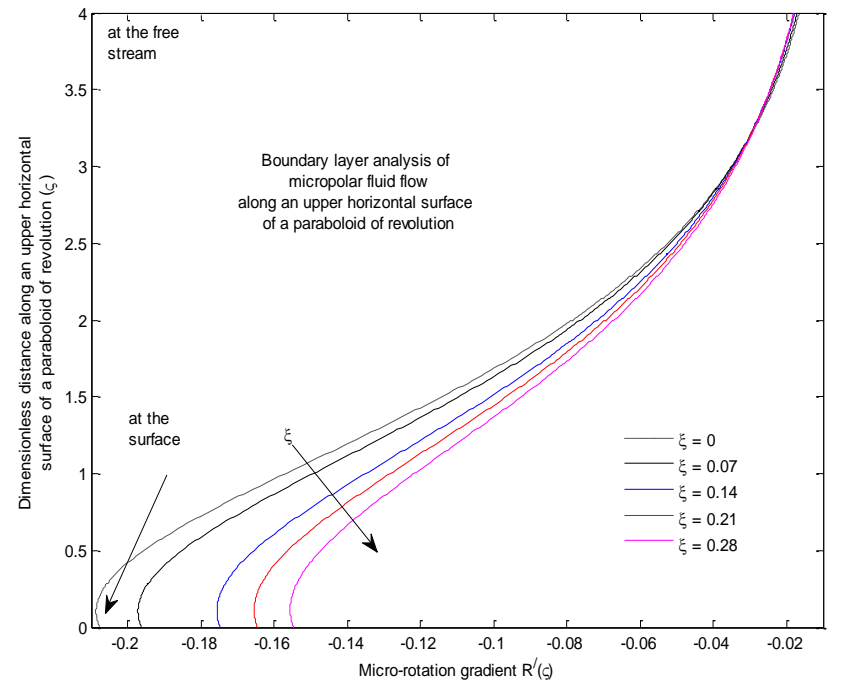

Fig. 7 Effect of $\xi$ on $R^{\prime}(\varsigma)$

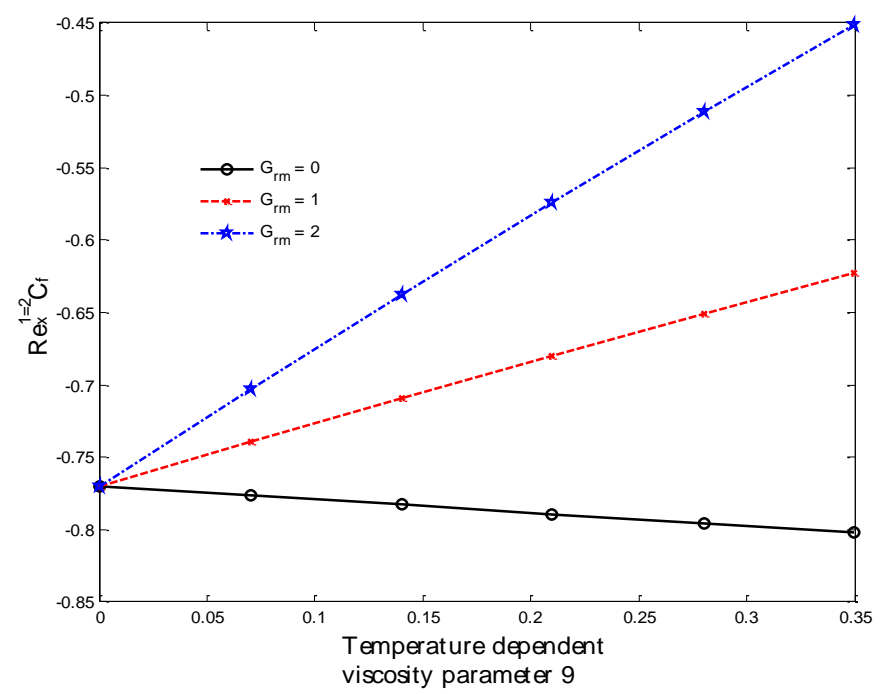

Fig. 8 Variations in $\operatorname{Re}_{x}^{1 / 2} C f$ with $\xi$ and $G_{r m}$

Table 1 Variation in $\mathrm{F}^{\prime \prime}(0)$ and $R(0)$ at various values of $\gamma, \xi$ and $\zeta$.

\begin{tabular}{l|cc} 
& $\mathrm{F}^{\prime \prime}(0) \quad \gamma=0$ & $R(0) \quad \gamma=0$ \\
\cline { 2 - 3 }$\xi=0.00, \zeta=1.5$ & -0.6977510522 & 0.3488755261 \\
$\xi=0.1, \zeta=1.5$ & -0.6656860156 & 0.3328430078 \\
\hline$\xi=0.2, \zeta=1.5$ & -0.6340845293 & 0.3170422646 \\
$\xi=0.1, \zeta=0$ & -0.5845149322 & 0.2922574661 \\
\hline$\xi=0.1, \zeta=1$ & -0.6406878025 & 0.3203439012 \\
$\xi=0.1, \zeta=2$ & -0.6873842024 & 0.3436921012 \\
\hline & $\mathrm{F}^{\prime \prime}(0) \quad \gamma=3$ & $R(0) \gamma=3$ \\
$\xi=0.00, \zeta=1.5$ & -0.7306111337 & 0.3653055668 \\
\hline$\xi=0.1, \zeta=1.5$ & -0.6672116810 & 0.3336058405 \\
$\xi=0.2, \zeta=1.5$ & -0.6065322441 & 0.3032661220 \\
\hline$\xi=0.1, \zeta=0$ & -0.5673573988 & 0.2836786994 \\
$\xi=0.1, \zeta=1$ & -0.6387261927 & 0.3193630963 \\
\hline$\xi=0.1, \zeta=2$ & -0.6896078297 & 0.3448039148
\end{tabular}




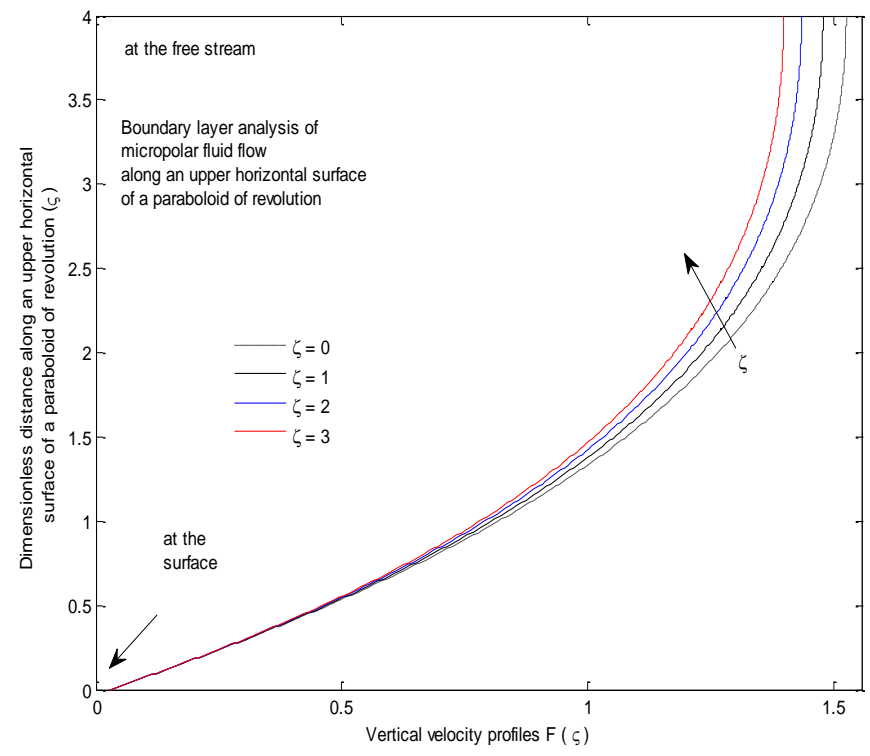

Fig. 9 Effect of $\zeta$ on $F(\varsigma)$

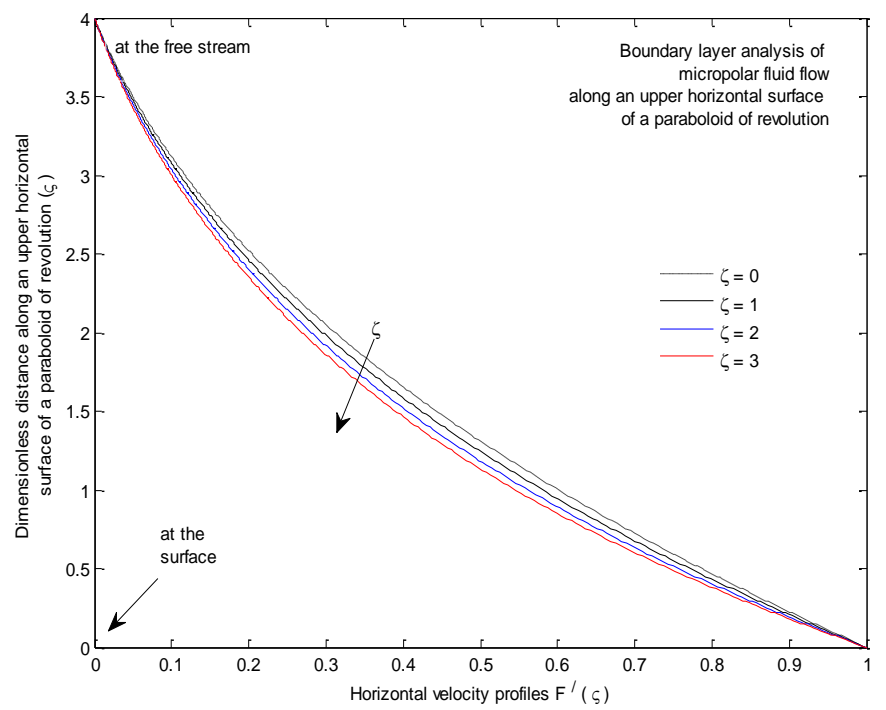

Fig. 10 Effect of $\zeta$ on $F^{\prime}(\varsigma)$

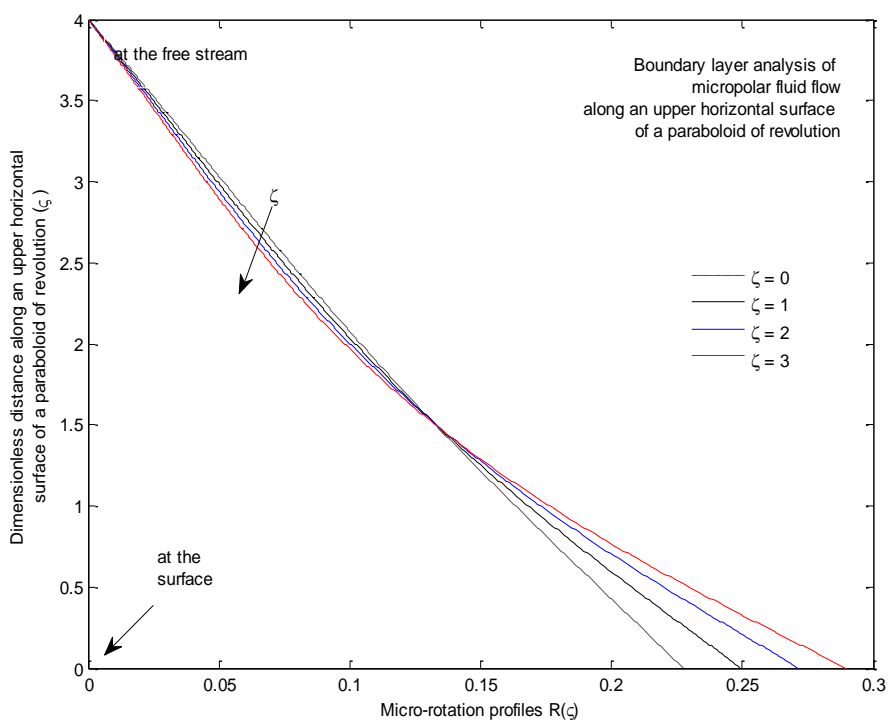

Fig. 11 Effect of $\zeta$ on $R(\varsigma)$

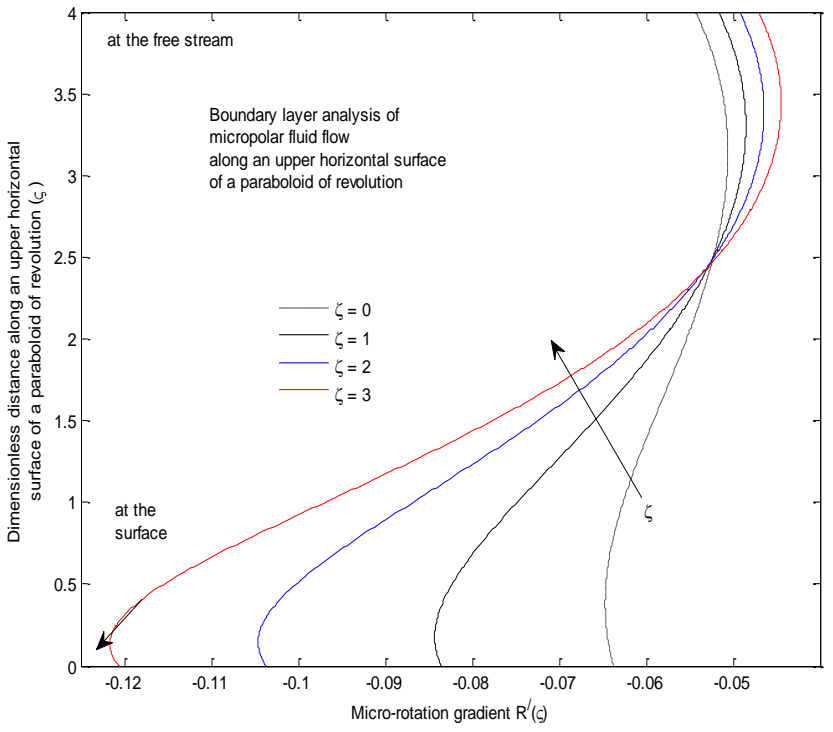

Fig. 12 Effect of $\zeta$ on $R^{\prime}(\varsigma)$

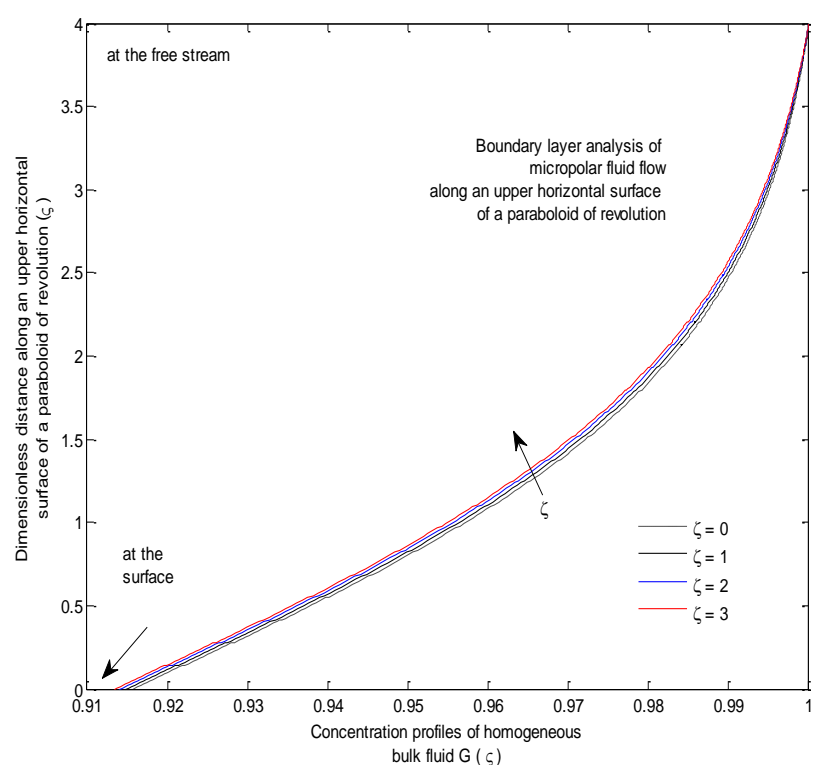

Fig. 13 Effect of $\zeta$ on $G(\varsigma)$

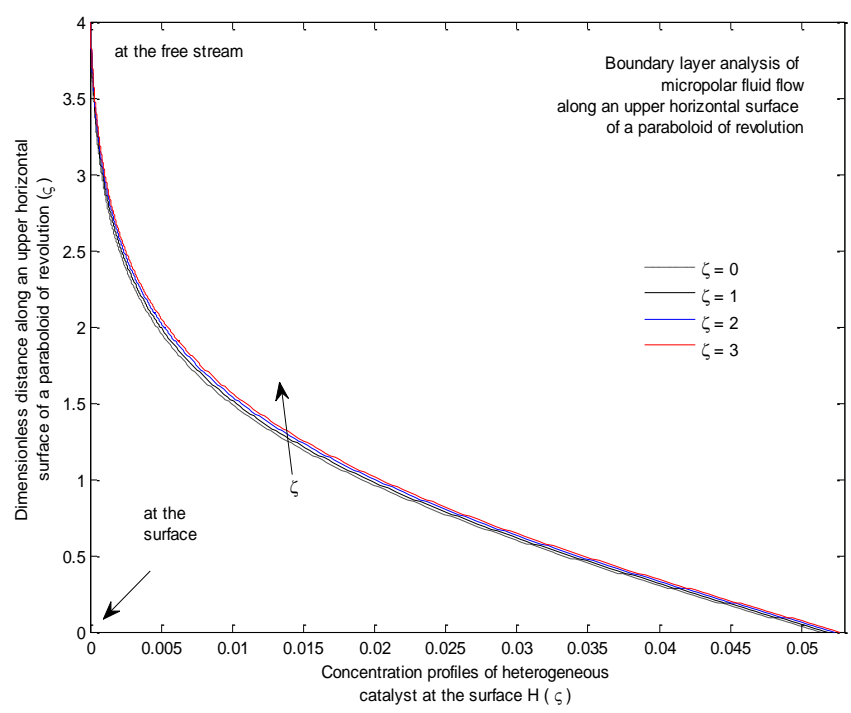

Fig. 14 Effect of $\zeta$ on $H(\varsigma)$ 


\subsection{Effects of micro-rotation parameter ( $)$ on the flow of micropolar fluid within boundary layer}

Using $\xi=0.2, \quad \aleph=0.4, m=0.3, \quad \varepsilon=0.2, \operatorname{Pr}=0.7, \quad S_{c a}=0.62$, $S_{c b}=1.3, R=0.1, \theta_{w}=0.2, m_{o}=0.5, G_{r m}=1, K=0.05, \gamma=0.2$,

$\mathrm{n}=0.3, \zeta=0.1, \chi=0.05, \Lambda=0.05, \delta=1.2$ and $N=3$ effects of $\aleph$ are illustrated in Fig. 17 - Fig. 23. In this investigation, the magnitude of $\aleph$ within the interval of 0 and 4 is considered. The vertical velocity and horizontal velocity profiles increase with an increase in the magnitude of $\aleph$, see Fig. 17 and Fig. 18. Also, it is confirmed that shear stress profiles near an uhspr increases and decreases near the free stream as shown in Fig. 19. Also, a negligible effect of $\aleph$ is observed on temperature and temperature gradient profiles. These figures are not presented here for brevity. Near the upper horizontal surface of a paraboloid of revolution, it is observed that micro-rotation of particles $R(\varsigma)$ in the fluid decreases while micro-rotation gradient $R^{\prime}(\varsigma)$ increase within the same interval near an uhspr; see Fig. 20 and Fig. 21. At the quartic autocatalytic level of chemical reaction $(N=3)$, it is observed that concentration of homogeneous bulk fluid increases while the concentration of heterogeneous catalyst at the surface decreases with $\aleph$; see Fig. 22 and Fig. 23.

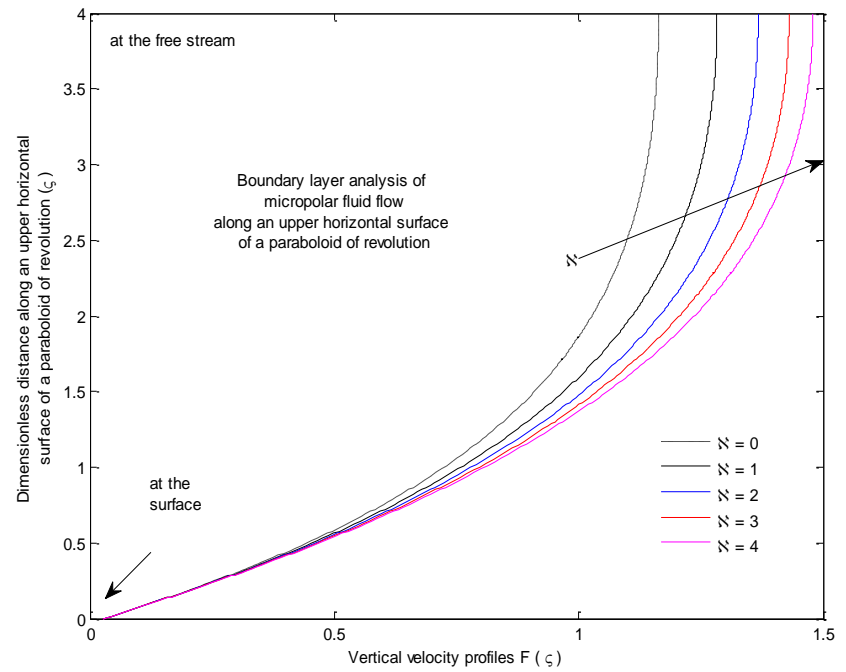

Fig. 15 Effect of ぶ on $F(\varsigma)$

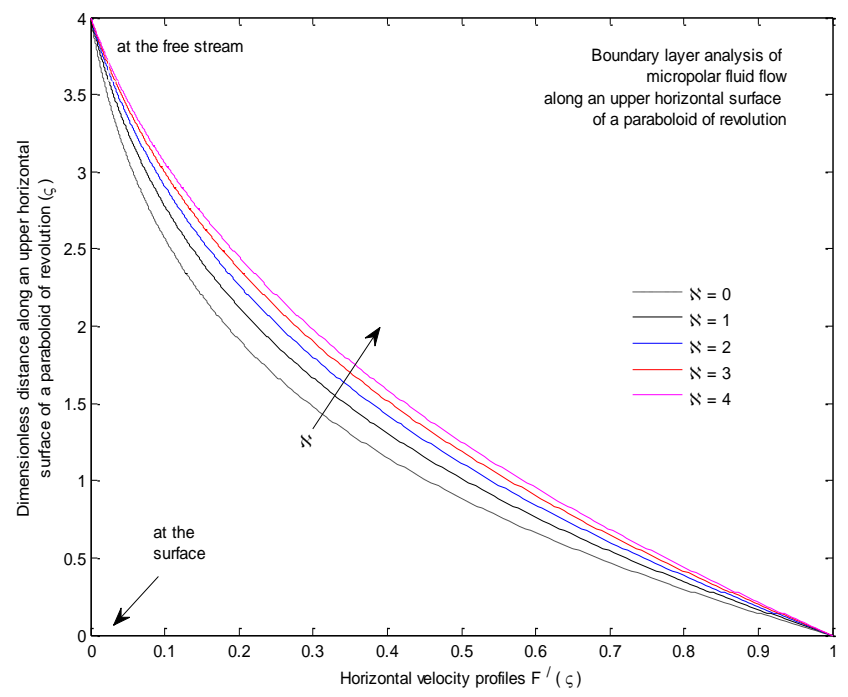

Fig. 16 Effect of $\aleph$ on $F^{\prime}(\varsigma)$

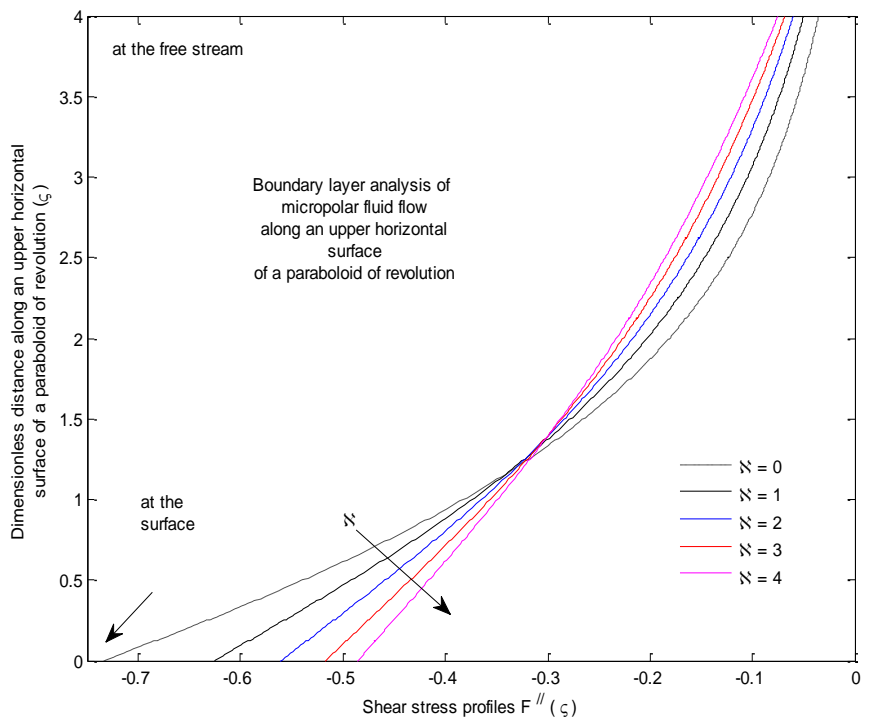

Fig. 17 Effect of $\aleph$ on $F^{\prime \prime}(\varsigma)$

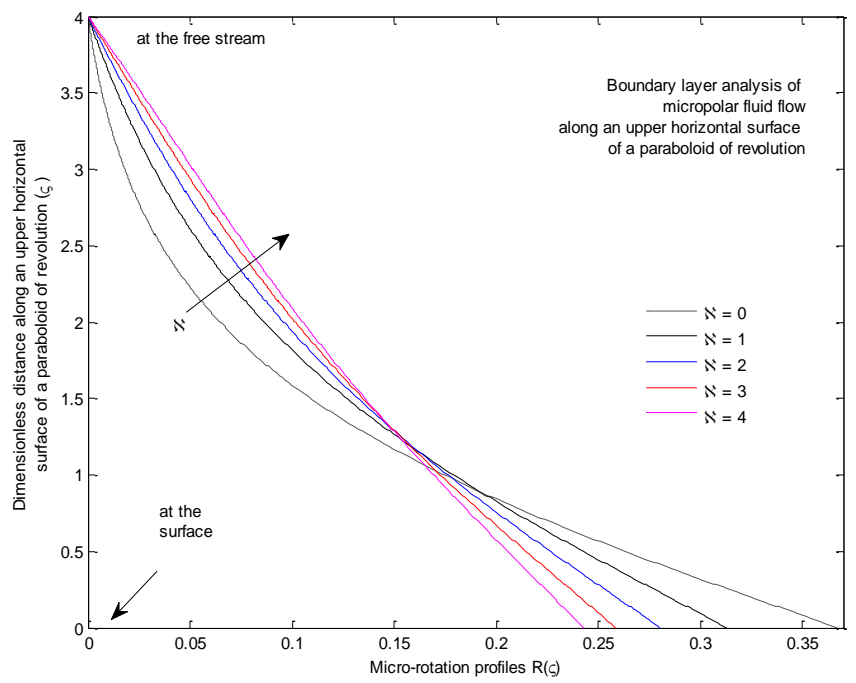

Fig. 18 Effect of $\aleph$ on $R(\varsigma)$

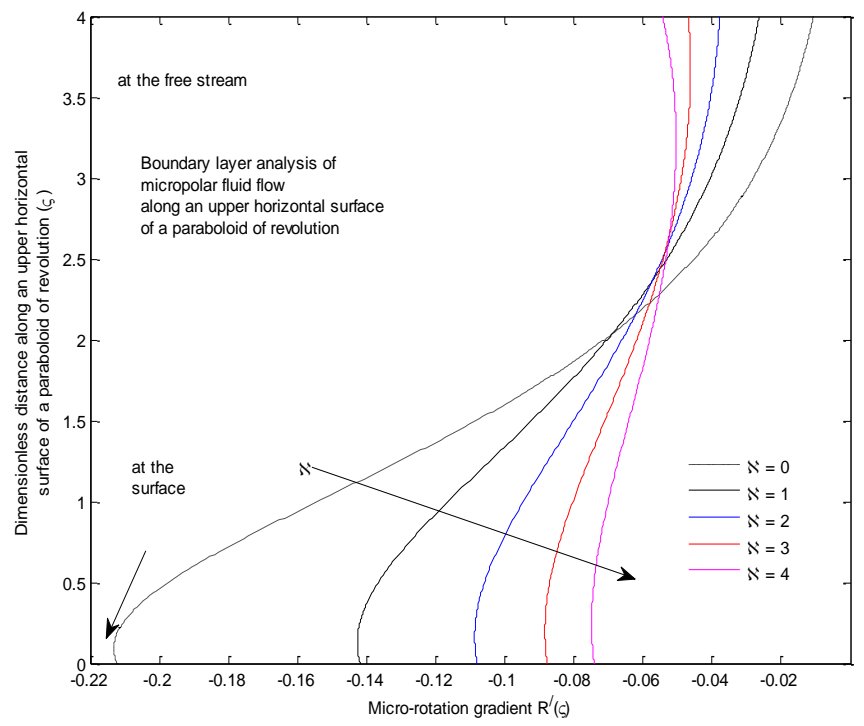

Fig. 19 Effect of $\aleph$ on $R^{\prime}(\varsigma)$ 


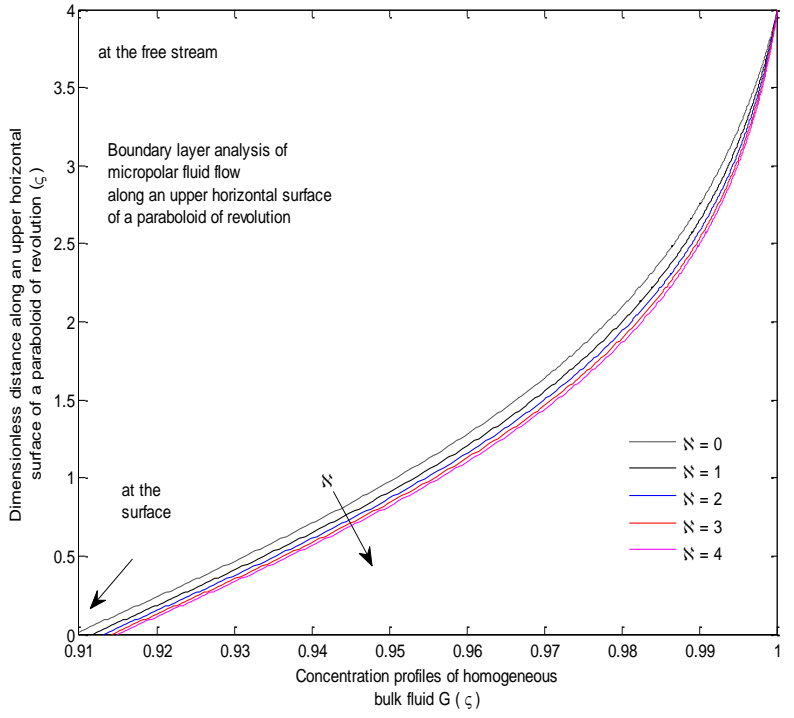

Fig. 20 Effect of ぶ on $G(\varsigma)$

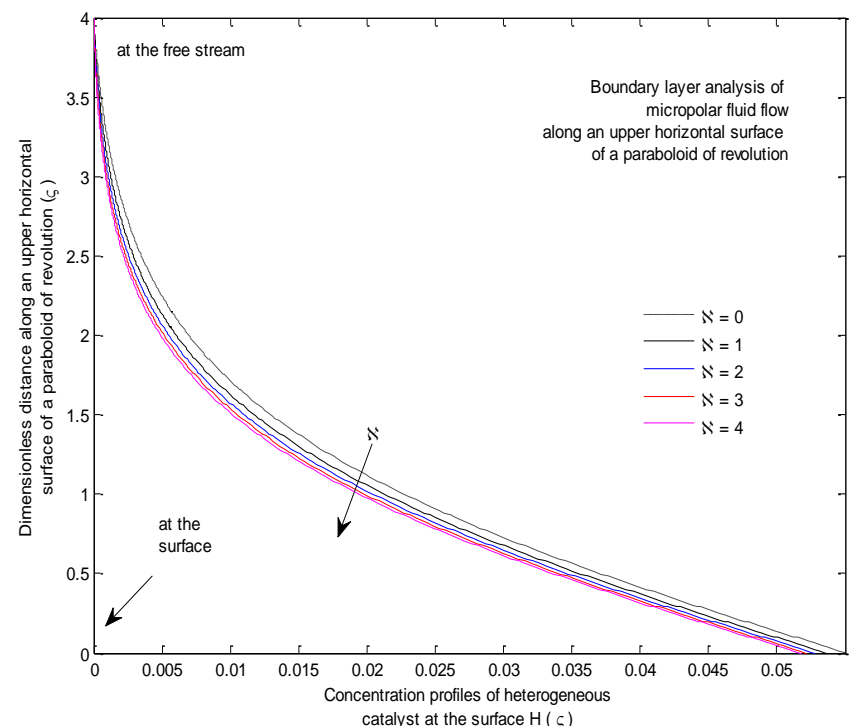

Fig. 21 Effect of $\aleph$ on $H(\varsigma)$

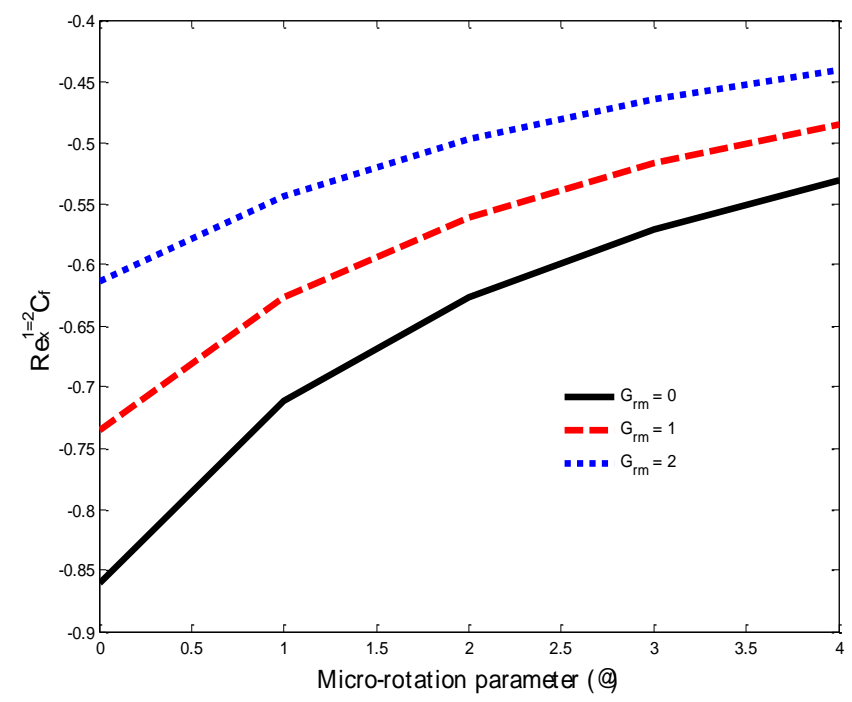

Fig. 22 Variations in $\operatorname{Re}_{x}^{1 / 2} C f$ with $\aleph$ and $G_{r m}$

\subsection{Effects of nonlinear thermal radiation parameter $\left(R_{a}\right)$ and thickness parameter $\chi$ on the flow of micropolar fluid within boundary layer formed on $u h s p r$}

When $\quad \xi=0.1, \quad \zeta=0.8, \aleph=2, \quad m=0.3, \quad \varepsilon=0.2, \operatorname{Pr}=0.7$, $S_{c a}=0.62, S_{c b}=1.3, R=0.1, \theta_{w}=1.2, m_{o}=0.5, G_{r m}=1, K=0.05$, $\gamma=0.2, \mathrm{n}=0.3, \zeta=0.1, \chi=0.05, \Lambda=0.05, \delta=1.2 \quad$ and $\quad N=3$ at various values of thermal radiation parameter $R_{a}$ when the magnitude of thickness parameter $\chi$ is small and large are illustrated in Fig. 25 Fig. 28. It is worth noteworthy that the temperature function $\Theta(\varsigma)$ depicts a decrement with the increasing $R_{a}$. Furthermore, the rate of decrease is more enhanced when the magnitude of thickness parameter is large. Physically, as the magnitude of $\chi$ increases, this corresponds to different objects of a paraboloid of revolution with higher thickness. In view of this, more heat is lost during when the thickness is large; see Fig. 25 and Fig. 26. The influence of nonlinear thermal radiation parameter $R_{a}$ on temperature gradient is shown in Fig. 27 and Fig. 28 at two different values of thickness parameter. It is noticed that Nusselt number $\mathrm{Nu}_{x} \mathrm{Re}_{x}^{1 / 2}$ which is proportional to local heat transfer rate decreases with $R_{a}$. However, it is seen that the nature of local heat transfer rate within the fluid layer near a free stream can be controlled by using $\chi$.

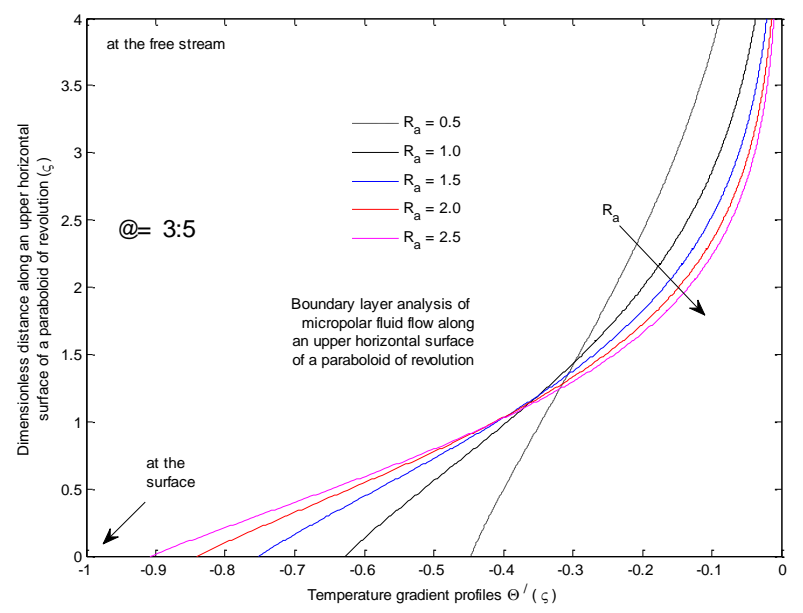

Fig. 23 Effect of $R_{a}$ on $\Theta(\varsigma)$ when $\chi=0.05$

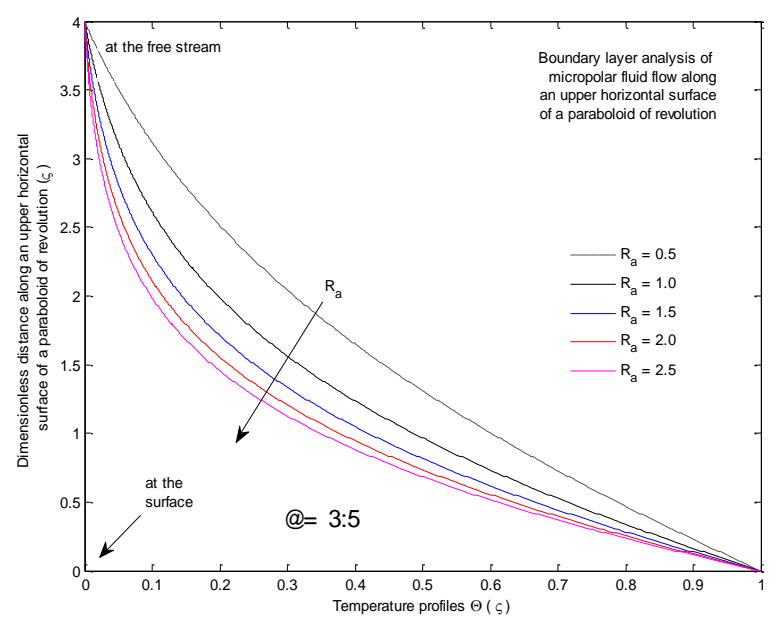

Fig. 24 Effect of $R_{a}$ on $\Theta(\varsigma)$ when $\chi=3.5$ 


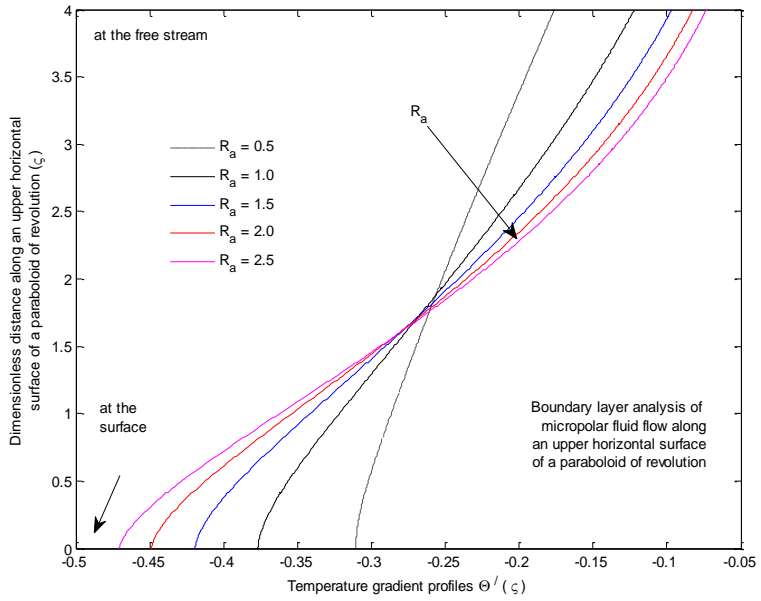

Fig. 25 Effect of $R_{a}$ on $\Theta^{\prime}(\varsigma)$ when $\chi=0.05$

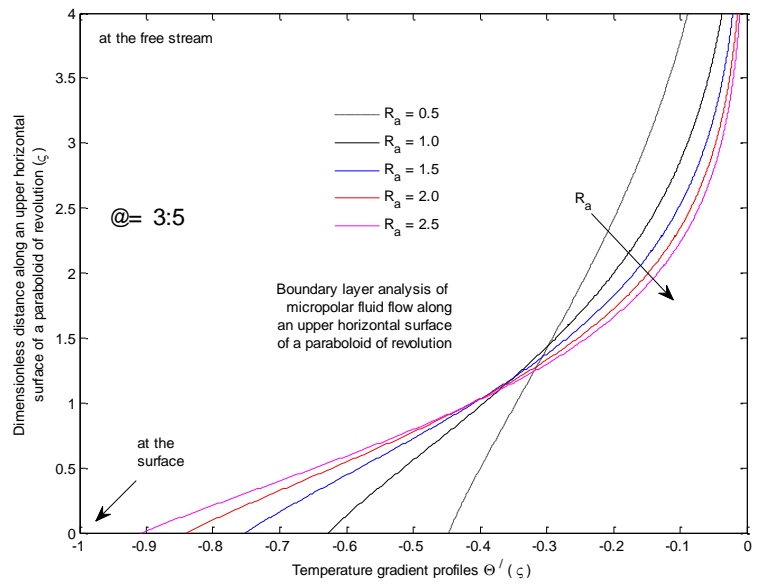

Fig. 26 Effect of $R_{a}$ on $\Theta^{\prime}(\varsigma)$ when $\chi=3.5$

\section{CONCLUSION}

This paper presents the nature of skin friction coefficient, heat transfer rate, velocity, temperature, the concentration of homogeneous bulk fluid (i.e. air in which tiny particle rotates) and heterogeneous catalyst on a stretchable surface next to the object called paraboloid of revolution. In this report, the dynamic viscosity and thermal conductivity are assumed to vary linearly with temperature while vortex viscosity varies exponentially with temperature. Suitable similarity transformation for scaling angular momentum is introduced. The results show that

i. The proposed similarity variable successfully nondimensionalized and parameterized the angular momentum for boundary layer flow past $u$ hspr.

ii. Temperature dependent dynamic viscosity parameter increases vertical velocity near a free stream but reduces micro-rotation near $u$ hspr.

iii. Minimum $\operatorname{Re}_{x}^{1 / 2} C f$ is guaranty at any values of buoyancy parameter when dynamic viscosity of the micropolar fluid is treated as a constant function of temperature.

iv. Micro-rotation of tiny particle which rotates in air as it flow past a pointed surface of a rocket (as in space science), over a bonnet of a car (as in automobile) and past a pointed surface of aircraft is a decreasing function of vertical velocity near free stream with an increase in the magnitude of temperature dependent vortex viscosity. Opposite effect of micro-rotation parameter on vertical velocity is certain.

v. Effects of thermal radiation parameter on temperature profile and heat transfer rate can be greatly influenced by thickness parameter.

\section{NOMENCLATURE}

$\begin{array}{ll}x & \text { dimensional coordinate on } u h s p r(\mathrm{~m}) \\ y & \text { vertical coordinate }(\mathrm{m}) \\ u & \text { velocity component along the } \mathrm{x} \text {-direction }\left(\mathrm{ms}^{2}\right) \\ v & \text { velocity component along the y-direction }\left(\mathrm{ms}^{2}\right) \\ P & \text { dimensional angular velocity } \\ j & \text { micro-inertia density } \\ T_{w}(x) & \text { wall temperature } \\ T_{\infty} & \text { free stream temperature } \\ g & \text { acceleration due to gravity } \\ \tau_{w} & \text { shear stress } \\ c_{p} & \text { specific heat at constant pressure } \\ G r e e k \text { Symbols } \\ \xi & \text { temperature dependent dynamic viscosity parameter } \\ \zeta & \text { temperature dependent vortex viscosity parameter } \\ \varsigma & \text { dimensionless distance on } u h s p r \\ \rho & \text { density of the micropolar fluid }\left(\mathrm{kg} / \mathrm{m}^{3}\right) \\ \sigma & \text { Stefan-Boltzmann constant }\left(\mathrm{W} / \mathrm{m}^{2} \cdot \mathrm{K}^{4}\right) \\ \mu & \text { dynamic viscosity of the micropolar fluid } \\ \mu^{*} & \text { dynamic viscosity at free stream } \\ \beta & \text { volumetric thermal-expansion coefficient } \\ \delta & \text { boundary layer thickness } \\ \psi(x, y) & \text { stream function } \\ \gamma^{*} & \text { spin gradient viscosity of the micropolar fluid } \\ \tau & \text { vortex viscosity of the micropolar fluid } \\ \tau^{*} & \text { vortex viscosity at free stream } \\ \kappa & \text { thermal conductivity of the micropolar fluid } \\ & \end{array}$

\section{REFERENCES}

Animasaun, I. L. 2015, "Double-Diffusive Unsteady Convective Micropolar Flow Past a Vertical Porous Plate Moving through Binary Mixture Using Modified Boussinesq Approximation," Ain Shams Engineering Journal, 7(2), 755 - 765. http://dx.doi.org/10.1016/j.asej.2015.06.010

Animasaun, I. L. 2015, "Dynamics of Unsteady MHD Convective Flow With Thermophoresis of Particles And Variable Thermo-Physical Properties Past a Vertical Surface Moving Through Binary Mixture," Open Journal of Fluid Dynamics, 5(2), 106 - 120 http://dx.doi.org/10.4236/ojfd.2015.52013

Animasaun, I. L. 2016, “47nm Alumina-Water Nanofluid Flow Within Boundary Layer Formed on Upper Horizontal Surface of Paraboloid of Revolution in the Presence of Quartic Autocatalysis Chemical Reaction," Alexandria Engineering Journal, 55(3), 2375-2389. http://dx.doi.org/10.1016/j.aej.2016.04.030

Animasaun, I. L. 2016, “Melting Heat And Mass Transfer in Stagnation Point Micropolar Fluid Flow of Temperature Dependent Fluid 
Viscosity And Thermal Conductivity at Constant Vortex Viscosity," Journal of the Egyptian Mathematical Society, 25(1), 79 - 85. http://dx.doi.org/10.1016/j.joems.2016.06.007.

Animasaun, I. L., Raju, C. S. K. and Sandeep, N. 2016, "Unequal Diffusivities Case of Homogeneous-Heterogeneous Reactions Within Viscoelastic Fluid Flow in The Presence Of Induced Magnetic-Field And Nonlinear Thermal Radiation," Alexandria Engineering Journal, $\mathbf{5 5}(2), 1595-1606$.

http://dx.doi.org/10.1016/j.aej.2016.01.018

Babu, M.J., Gupta, R. and Sandeep, N. 2015, "Effect of Radiation and Viscous Dissipation on Stagnation-Point Flow of a Micropolar Fluid Over a Nonlinearly Stretching Surface with Suction/Injection," Journal of Basic and Applied Research International, 7(2), 73-82

Blasius, H., 1908, "Grenzschichten in Flussigkeiten mit kleiner Reibung,” Z. Math. Phys. 56, 1-37.

Charraudeau, J. 1975, "Influence De Gradients De Properties Physiques En Convection Force Application Au Cas Du Tube," International Journal of Heat and Mass Transfer, 18, 87 - 95. http://dx.doi.org/10.1016/0017-9310(75)90011-3

Chaudhary, M. A. and Merkin, J. H. 1995, "A Simple Isothermal Model For Homogeneous-Heterogeneous Reactions in Boundary-Layer Flow. II Different Diffusivities for Reactant and Autocatalyst," Fluid Dynamics Research, 16(6), 335 - 359.

http://dx.doi.org/10.1016/0169-5983(95)90813-h

Chaudhary, M. A. and Merkin J. H. 1995, “A Simple Isothermal Model for Homogeneous-Heterogeneous Reactions in Boundary-Layer Flow. I Equal Diffusivities," Fluid Dynamics Research 16(6), 311 - 333. http://dx.doi.org/10.1016/0169-5983(95)00015-6

Cortell, R. 2014, "Fluid Flow and Radiative Nonlinear Heat Transfer over Stretching Sheet," Journal of King Saud University-Science, 26(2), $161-167$.

http://dx.doi.org/10.1016/j.jksus.2013.08.004

Eringen, A.C. 1964, "Simple Microfluids," International Journal of Engineering Science, 2(2), 205 - 217.

http://dx.doi.org/10.1016/0020-7225(64)90005-9

Eringen, A.C. 1966, "Theory of Anisotropic Micropolar Fluids," International Journal of Engineering Science, 18(1), 5-17.

http://dx.doi.org/10.1016/0020-7225(80)90003-8

Gökhan, F. S. 2011, "Effect of the Guess Function \& Continuation Method on the Run Time of MATLAB BVP Solvers," Clara M., Ionescu (Ed.), 1. http://dx.doi.org/10.5772/19444

Gupta, D. 2014, "Non-Linear Problems in Micropolar Fluid Flows, Doctoral Dissertation," Jaypee Institute of Information Technology, Uttar Pradesh, India.

Hayat, T., Imtiaz, M., Alsaedi, A. and Kutbi, M. A. 2015, "MHD Three-Dimensional Flow of Nanofluid with Velocity Slip and Nonlinear Thermal Radiation," Journal of Magnetism and Magnetic Materials. 396, 31 - 37.

http://dx.doi.org/10.1016/j.jmmm.2015.07.091

Kierzenka, J. and Shampine, L. F. 2001, "A BVP Solver Based on Residual Control And The MATLAB PSE," ACM Transactions on Mathematical Software (TOMS), 27(3), 299-316. http://dx.doi.org/10.1145/502800.502801
Kumaran, G., Sandeep N. and I. L. Animasaun 2017, "Computational Modeling of Magnetohydrodynamic Non-Newtonian Fluid Flow Past a Paraboloid of Revolution, Alexandria Engineering Journal," in-press, http://dx.doi.org/10.1016/j.aej.2017.03.019

Lukazewicz, G. 1999, "Micropolar Fluids: Theory and Applications," Basel: Birkhauser, Boston. ISBN 978-0-8176-4008-8 http://dx.doi.org/10.1007/978-1-4612-0641-5

Lynch, D.T. 1992, "Chaotic Behavior of Reaction Systems: Mixed Cubic and Quadratic Autocatalysis," Chemical Engineering Science, 47(17/18), 4435 - 4444. http://dx.doi.org/10.1016/0009-2509(92)85121-q

Makinde, O.D. and Animasaun, I.L. 2016a, "Bioconvection in MHD Nanofluid Flow with Nonlinear Thermal Radiation and Quartic Autocatalysis Chemical Reaction Past an Upper Surface of a Paraboloid of Revolution," International Journal of Thermal Sciences, 109, 159 171.

http://dx.doi.org/10.1016/j.ijthermalsci.2016.06.003

Makinde, O.D. and Animasaun, I.L. 2016b, "Thermophoresis and Brownian Motion Effects on MHD Bioconvection of Nanofluid with Nonlinear Thermal Radiation and Quartic Chemical Reaction Past an Upper Horizontal Surface of a Paraboloid of Revolution," Journal of Molecular liquids, 221, 733 - 743.

http://dx.doi.org/10.1016/j.molliq.2016.06.047

Mohantya, B., Mishraa, S.R. and Pattanayak, H.B. 2015, "Numerical Investigation on Heat And Mass Transfer Effect of Micropolar Fluid over a Stretching Sheet Through Porous Media," Alexandria Engineering Journal, 54(2), 223-232.

http://dx.doi.org/10.1016/j.aej.2015.03.010

Moore, D.W. 1963, "The Boundary Layer on a Spherical Gas Bubble," Journal of Fluid Mechanics, 16(2), 161. http://dx.doi.org/10.1017/S0022112063000665

Motsa, S. S. and Animasaun, I. L. 2016a, "Unsteady Boundary Layer Flow over a Vertical Surface due to Impulsive and Buoyancy In The Presence of Thermal-Diffusion And Diffusion-Thermo Using Bivariate Spectral Relaxation Method," Journal of Applied Fluid Mechanics, 9(5), 2605-2619.

Motsa, S. S. and Animasaun, I. L. 2016b, "Paired Quasi-Linearization Analysis of Heat Transfer in Unsteady Mixed Convection Nanofluid Containing Both Nanoparticles and Gyrotactic Microorganisms due to Impulsive Motion," Journal of Heat Transfer-ASME, 138(114503), 1-8. http://dx.doi.org/10.1115/1.4034039

Mukhopadhyay, S. 2009, "Effects of Radiation And Variable Fluid Viscosity on Flow and Heat Transfer along a Symmetric Wedge," Journal of Applied Fluid Mechanics, 2(2), 29 - 34.

Murphy, J. S., 1953, "Some Effects of Surface Curvature on Laminar Boundary-Layer Flow," Journal of the Aeronautical Sciences, 20(5), 338-344. http://dx.doi.org/10.2514/8.2638

Na, T.Y. 1979, "Computational Methods in Engineering Boundary Value Problems,” Academic Press, New York. ISBN 978-0125126502

Naramgari, S. and Sulochana, C. 2016, "MHD Flow Over a Permeable Stretching/Shrinking Sheet of a Nanofluid with Suction/Injection," Alexandria Engineering Journal, 55(2), 819 - 827 http://dx.doi.org/10.1016/j.aej.2016.02.001 
Ojjela, O. and Kumar, N. N. 2016, "Chemically Reacting Micropolar Fluid Flow And Heat Transfer Between Expanding or Contracting Walls With Ion Slip, Soret And Dufour Effects," Alexandria Engineering Journal, 55(2), 1683-1694. http://dx.doi.org/10.1016/j.aej.2016.02.026

Pantokratoras, A. 2009, "A Common Error Made in Investigation of Boundary Layer Flows," Applied Mathematical Modelling, 33(1), 413422.

http://dx.doi.org/10.1016/j.apm.2007.11.009

Perdikis, C. and Raptis, A. 1996, "Heat Transfer of a Micropolar Fluid by the Presence of Radiation," Heat Mass Transfer, 31(6), 381 - 382 http://dx.doi.org/10.1007/BF02172582

Rosseland, S. 1931, "Astrophysik aud atom-theoretische Grundlagen," Springer, Berlin, 41-44.

Sakiadis, B. C. 1961, "Boundary Layer Behavior on Continuous Solid Surfaces: The Boundary Layer on a Continuous Flat Surface," American Institute of Chemical Engineers (AIChE), 7(2), 221-225. http://dx.doi.org/10.1002/aic.690070211

Sandeep, N. and Animasaun I .L. (2017) Heat Transfer in Wall Jet Flow of Magnetic-Nanofluids with Variable Magnetic Field, Alexandria Engineering Journal, in-press. http://dx.doi.org/10.1016/j.aej.2016.12.019

Sandeep, N., Koriko, O. K. and Animasaun, I. L. 2016, "Modified Kinematic Viscosity Model For 3D-Casson Fluid Flow Within Boundary Layer Formed on a Surface At Absolute Zero," Journal of Molecular Liquids, 221, 1197 - 1206.

http://dx.doi.org/10.1016/j.molliq.2016.06.049

Sawchuk, S. P. and Zamir M. 1992, "Boundary Layer on a Circular Cylinder in Axial Flow," International Journal of Heat and Fluid Flow, 13(2), 184-188.

http://dx.doi.org/10.1016/0142-727x(92)90026-6

Sowerby, L. and Cooke, J., 1953, "The Flow of Fluid along Corners and Edges," The Quarterly Journal of Mechanics and Applied Mathematics, 6(1), 50 - 70.

http://dx.doi.org/10.1093/qjmam/6.1.50

Sulochana, C., Ashwinkumara, G. P. and Sandeep, N. 2016, "Transpiration Effect on Stagnation-Point Flow of a Carreau Nanofluid in the Presence of Thermophoresis and Brownian Motion," Alexandria Engineering Journal, 55(2), 1151 - 1157. http://dx.doi.org/10.1016/j.aej.2016.03.031 